\title{
Adaptive Robust Posture Control of a 3-RPS Pneumatic Parallel Platform with Unknown Deadzone
}

\author{
Guoliang Tao, ${ }^{1}$ Ce Shang, ${ }^{1}$ and Deyuan Meng ${ }^{2}$ \\ ${ }^{1}$ State Key Laboratory of Fluid Power and Mechatronic Systems, Zhejiang University, Hangzhou 310027, China \\ ${ }^{2}$ School of Mechatronic Engineering, China University of Mining and Technology, Xuzhou, Jiangsu 221116, China
}

Correspondence should be addressed to Ce Shang; czesh@zju.edu.cn

Received 7 January 2016; Accepted 28 April 2016

Academic Editor: Tarek Ahmed-Ali

Copyright (C) 2016 Guoliang Tao et al. This is an open access article distributed under the Creative Commons Attribution License, which permits unrestricted use, distribution, and reproduction in any medium, provided the original work is properly cited.

\begin{abstract}
An adaptive robust controller integrated with online deadzone estimation is proposed. This controller provides trajectory tracking control for pneumatic parallel mechanisms. Due to the air compressibility and nonlinear characteristics of the pneumatic system, unknown parameters in the model are selected to build online estimation matrices with the robust parts considered in the design. As each proportional valve has specific values of deadzone boundary points, the deadzone parts are integrated into the online estimator, and an inverse deadzone compensator is used to overcome nonlinear limitations. The effectiveness of the method was verified by simulation and experiment, and theoretical stability was demonstrated using the Lyapunov method. Experiments in an actual plant with the proposed controller indicated that the performance of the pneumatic platform can be as good as that of ideal deadzone inverse compensation. The deadzone estimated parameters converged to the real values quickly. Additionally, this algorithm was effective under a compound reference input trajectory; thus, the controller is expected to perform well in actual working situations.
\end{abstract}

\section{Introduction}

Parallel mechanisms are now used widely in many industrial and civil applications, such as motion simulators, adjustment platforms, and virtual reality equipment [1]. Compared with a full six-degrees-of-freedom (6-DoF) structure, such as a Stewart platform, an incomplete DoF 3-RPS (revolute, prismatic, and spherical) manipulator retains the three most important freedoms: roll, pitch, and heave. Many 3-RPS platforms that are used currently are driven by electric or hydraulic cylinders to provide adequate control precision [2]. Due to the compressibility of air and the nonlinear characteristics of pneumatic elements, pneumatic servo control is much more difficult to achieve than electric or hydraulic ones $[3,4]$. Although the trajectory tracking accuracy is much lower, for some special situations, the lower cost advantage of pneumatic cylinders provides incentive for use.

Researchers and engineers have been working on improvements in pneumatic servo control performance. Many studies have been performed in this area with many algorithms applied to pneumatic systems in recent years, such as feedback linearization [5], self-tuning [6], fuzzy [7], and sliding mode controls [8-10]. Based on the back-stepping method, the adaptive robust controller has attracted attention for its resistance to parameter uncertainties and nonlinear characteristics [11, 12]. Applying adaptive robust method aspects in controller design has been shown to be an effective way to raise pneumatic servo system position control performance for a single cylinder [13]. For parallel stages posture control driven by pneumatic cylinders, some achievements have been made with different control methods [14-16]. The precisions of such platforms driven by pneumatic cylinders in these studies are about $5-15 \%$ of the amplitudes. Compared with the hydraulic stage and electric stage that demonstrate better stiffness, higher accuracy, and a higher response frequency, commercial pneumatic parallel mechanism production is still in its infancy.

Deadzone properties are quite common in actuator models, especially in the proportional valves $[17,18]$. Figure 4 presents the typical deadzone mass-flow rate features of the FESTO MPYE 5-1/4-010-B proportional directional valve. When the control voltage is within about $4.3-5.7 \mathrm{~V}$, the output 

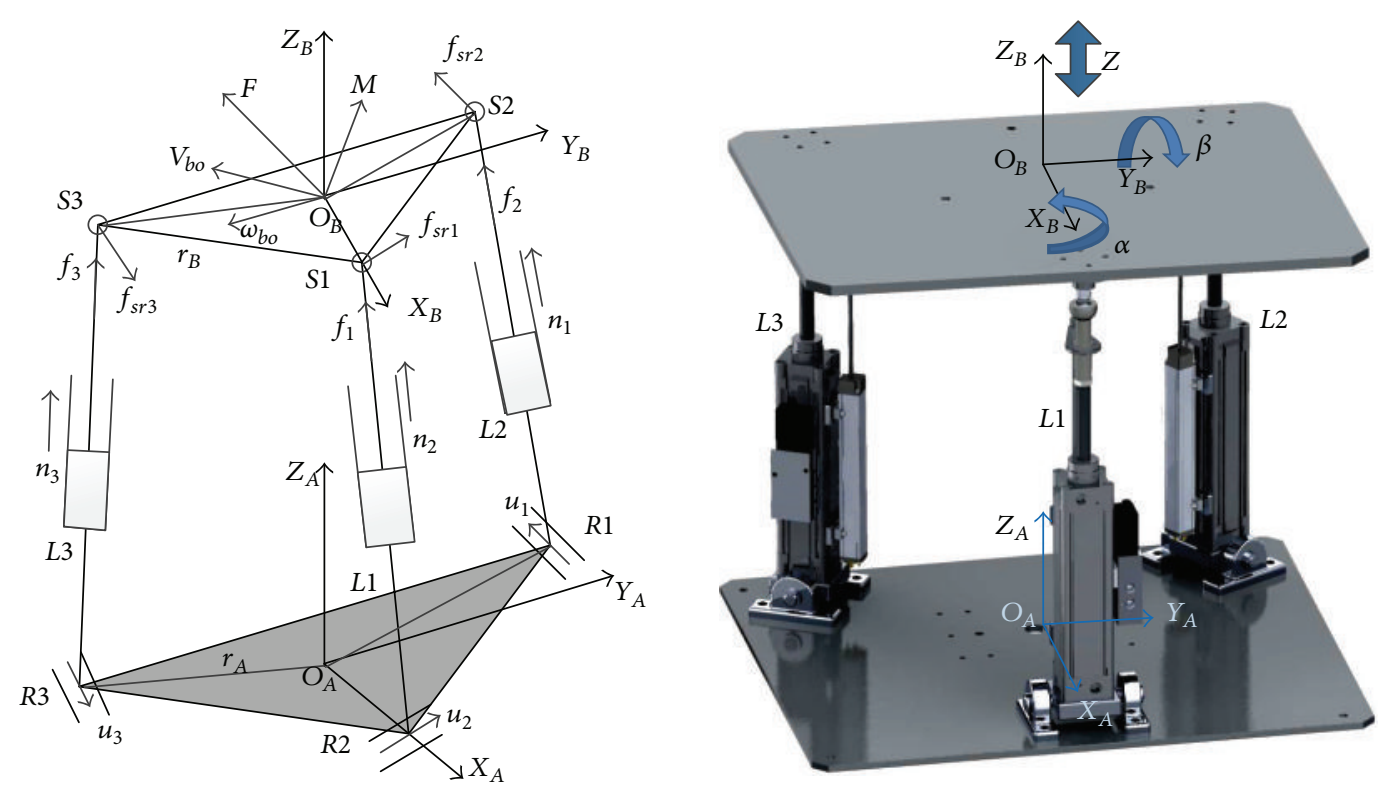

FIGURE 1: Mechanical structure of 3-RPS pneumatic platform.

mass flow will be almost zero for both paths. This feature can be compensated for by the inverse deadzone calculation as long as the deadzone boundary values are known definitely. Unfortunately, each valve has a specific deadzone property. The variation is about $0.2 \mathrm{~V}$ for valves from different manufacturing batches and about $0.07 \mathrm{~V}$ for valves from the same batch, which is caused by manufacturing processing and assembly tolerances. Experiments have shown that even with a slight error between the model and the actual plant control precision will be affected significantly $[19,20]$. Because the proposed algorithm is aimed at a universal controller, not for any one system in the laboratory, the boundary points cannot be set as constants in the controller for all the valves in the model $[21,22]$. Based on concepts of adaptive robust control (ARC) and online parameter estimation, the deadzone key values have to be considered in the unknown parameters vector to raise the posture precision and controllers adaptability [23]. Researchers have studied hydraulic and electric systems [24-26], but for pneumatic systems, it was typically considered as a constant value in the model and the inverse deadzone model was built to compensate for this nonlinear feature $[18,27]$. In recent research, deadzone online compensation has not been considered in parallel mechanism control to increase control performance.

In this study, a controller was designed for a pneumatic parallel system model, based on aspects of ARC. Stability was demonstrated numerically and the effectiveness was validated in simulations and experiments. Moreover, a controller adopting deadzone compensation is proposed for posture control for a parallel pneumatic manipulator. The adaptability of this control algorithm satisfies realistic working situations, and the controller has much better tracking performance. In the second section, kinematic and dynamic models of the 3-RPS platform are established with mechanical constraints. Online estimations of load, friction, and deadzone, based on a least-squares method and noncontinuous projection mapping, are proposed in Section 3.1. A fast dynamic compensator is introduced into the robust controller design, based on the dynamic model of the 3-RPS system with unknown deadzone parameters, as described in Section 3.2. Based on the modeling and controller design, Section 4 provides experimental results, confirming the effectiveness of ARC with deadzone compensation, such that the improved algorithm makes the pneumatic platform less susceptible to deadzone variation effects as well as strengthens the adaptability of the controller. A summary and discussion are provided in Section 5. Proof of stability of the controller is set out in the appendix.

\section{Modeling of 3-RPS Pneumatic Platform}

2.1. Kinematics and Dynamics Analysis. The transition matrix is shown in the form of Tait-Bryan angles in (1), where $\mathrm{s}$ represents $\sin$ and $\mathrm{c}$ represents cos. $\left({ }^{A} X_{b o},{ }^{A} Y_{b o},{ }^{A} Z_{b o}\right)$ is the origin point location of the moving board in the fixed board coordinate. $\mathbf{u}_{i}$ is the normalized vector of the rotational joint axis. $\mathbf{n}_{i}$ is the normalized vector of $\mathbf{L}_{i}$. Figure 1 shows the mechanical structure and the coordinate configurations. Figure 2 shows the physical platform connected to the signal conditioning board and NI-PXI controller:

$$
{ }_{B}^{A} R=\left[\begin{array}{ccc}
c \beta c \gamma & -c \beta s \gamma & s \beta \\
c \alpha s \gamma+s \alpha s \beta c \gamma & c \alpha c \gamma-s \alpha s \beta s \gamma & -s \alpha c \beta \\
s \alpha s \gamma-c \alpha s \beta c \gamma & c \gamma s \alpha+c \alpha s \beta s \gamma & c \alpha c \beta
\end{array}\right] .
$$

$r_{A}$ and $r_{B}$ are the lengths of $O_{A} A_{i}$ and $O_{B} B_{i}$, respectively. In the current structure, the joint points $A_{i}$ and $B_{i}$ are all located on the same radius circle on each plane, and $O_{A}$ and $O_{B}$ are the centroids of the equilateral triangles $A_{1} A_{2} A_{3}$ and 


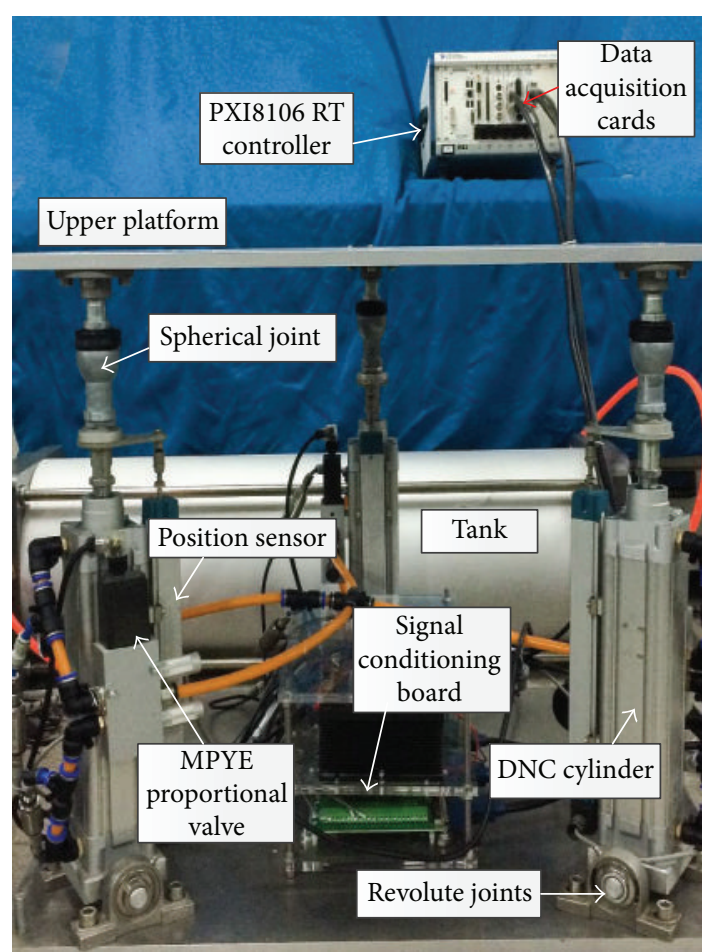

Figure 2: Physical 3-RPS pneumatic parallel platform.

$B_{1} B_{2} B_{3}$, respectively. Points $A_{i}, B_{i}, O_{A}$, and $O_{B}$ are in the same plane.

According to the mechanical structure, the constraint equations can be written as

$$
\begin{aligned}
& { }^{A} Y_{b o}=r_{B}(\cos \beta \sin \gamma), \\
& { }^{A} X_{b o} \\
& \quad=\frac{r_{B}}{2}(\cos \beta \cos \gamma+\sin \alpha \sin \beta \sin \gamma-\cos \alpha \cos \gamma), \\
& \gamma=-\arctan \frac{\sin \alpha \sin \beta}{\cos \alpha+\cos \beta} .
\end{aligned}
$$

In working space, when the desired trajectory $\mathbf{X}_{d}\{\alpha(t), \beta(t), Z(t)\}$ is given, the lengths of all actuators can be calculated in vector form, as shown in (3) according to the coordinate definition in Figure 1 and the constraints in (2):

$$
\mathbf{L}_{i}={ }_{B}^{A} T^{B} \mathbf{P}_{s i}-{ }^{A} \mathbf{P}_{R i}={ }_{B}^{A} R_{x y z}{ }^{B} \mathbf{P}_{s i}+{ }^{A} \mathbf{P}_{b o}-{ }^{A} \mathbf{P}_{R i},
$$

where $\mathbf{L}_{i}$ is the vector of each cylinder piston rod's direction in the fixed coordinate, $l_{i}=\left|\mathbf{L}_{i}\right|-L_{0 i}, L_{0 i}$ is the original length of the cylinder $(i)$, and $l_{i}$ is the actual position of each piston rod. Based on the discussion above, the transition functions from work space $\mathbf{X}_{d}(\alpha, \beta, z)$ to joint space $\mathbf{x}_{d}\left(l_{1}, l_{2}, l_{3}\right)$ are established.

Based on the structure of the 3-RPS pneumatic platform, the dynamic model in joint space can be written as follows:

$$
\mathbf{M}_{e} \ddot{\mathbf{x}}=\bar{A}_{a} \mathbf{p}_{L}-\mathbf{b} \dot{\mathbf{x}}-\mathbf{A}_{f} \mathbf{S}_{f}(\dot{\mathbf{x}})-\mathbf{F}_{L}+\mathbf{f}_{n}+\widetilde{\mathbf{f}}_{0},
$$

where $\bar{A}_{a}=H_{p} A_{a}, \mathbf{M}_{e}=\operatorname{diag}\left\{m_{1}, m_{2}, m_{3}\right\}, m_{i}$ stands for the equivalent inertial load of each piston $\operatorname{rod}, \mathbf{p}_{L}=$ $\left[p_{L 1}, p_{L 2}, p_{L 3}\right]^{T}, p_{L i}=p_{i a}-k_{i \text { area }} p_{i b}$, and $k_{\text {iarea }}$ is the area factor for the cylinder chambers within the piston rod. $\mathbf{x}=$ $\left[x_{1}, x_{2}, x_{3}\right]^{T}$ is the desired position curve of each cylinder. $\mathbf{p}_{L}$ is the first-level virtual input. The physical meaning of $p_{L i}$ is the equivalent pressure difference between the two chambers of one cylinder. $\widetilde{\mathbf{f}}_{n}=\left[\widetilde{f}_{n 1}, \widetilde{f}_{n 2}, \widetilde{f}_{n 3}\right]^{T}$ and $\widetilde{\mathbf{f}}_{0}=$ $\left[\tilde{f}_{01}, \tilde{f}_{02}, \tilde{f}_{03}\right]^{T}$ represent the modeling error and disturbance on each axis. $H_{p}$ is constant at $10^{5}$.

For the thermodynamic process in the cylinder chambers, the model of this system can be written as

$$
\begin{aligned}
\dot{\mathbf{p}}_{L}= & \mathbf{q}_{L}+\mathbf{F}_{p}+\mathbf{d}_{n}+\tilde{\mathbf{d}}_{0} \\
q_{L i}= & \frac{\gamma_{a} R}{H_{p} V_{i a}}\left(\dot{m}_{i a \text { in }} T_{s}-\dot{m}_{i a \mathrm{out}} T_{i a}\right) \\
& -\frac{\gamma_{a} R}{H_{p} V_{i b}}\left(\dot{m}_{i b \text { in }} T_{s}-\dot{m}_{i b o u t} T_{i b}\right) \\
F_{p i}= & -\frac{\gamma_{a} A_{i a}}{V_{i a}} \dot{x}_{i} p_{i a}-\frac{\gamma_{a} A_{i b}}{V_{i b}} \dot{x}_{i} p_{i b}+\frac{\gamma_{a}-1}{H_{p} V_{i a}} \dot{Q}_{i a} \\
& -\frac{\gamma_{a}-1}{H_{p} V_{i b}} \dot{Q}_{i b} .
\end{aligned}
$$

$\mathbf{q}_{L}=\left[q_{L 1}, q_{L 2}, q_{L 3}\right]^{T}$, which stands for the second-level virtual input, is used as the desired flow rate to calculate the control voltages of the proportional valves. $\mathbf{F}_{p}=\left[F_{p 1}, F_{p 2}, F_{p 3}\right]^{T}$ is the load matrix applied to cylinder piston rods. $\mathbf{d}_{n}=$ $\left[d_{n 1}, d_{n 2}, d_{n 3}\right]^{T}$ and $\tilde{\mathbf{d}}_{0}=\left[d_{01}, d_{02}, d_{03}\right]^{T}$ are the influence caused by the valve model and disturbance of air flow, respectively. $\tilde{\mathbf{f}}_{0}$ and $\tilde{\mathbf{d}}_{0}$ are all the nonlinear uncertainty parts in the model. $\gamma_{a}$ is the heat capacity ratio, $R$ is the ideal gas constant, $h$ is the thermal conductivity between air and inner chamber surface, and $p_{s}$ is the source pressure. The heat transfer between chamber and outside environment is $\dot{Q}_{i}=h S_{h i}(x)\left(T_{s}-T_{i}\right)$, where $S_{h i}(x)=2 A_{i}+\pi D(L / 2+x)$ is the area of heat exchange. The dynamic features of the valve core can be ignored, because the natural frequency of the proportional directional control valve (FESTO MPYE series) is much higher than the bandwidth of pneumatic servo system.

2.2. Deadzone Characteristics. Figure 3 gives the typical deadzone model in the mechatronics system. $u_{+}$and $u_{-}$ are the upper and lower bound points of the deadzone, respectively. $k_{x}$ is the slope of the output characteristic curve nearing the boundary point of $u_{+}$. Experiments showed that the features of the flow rate of the valve are symmetric; as such, the following equation can be used to describe the deadzone features:

$$
x_{e}=D(u)= \begin{cases}k_{x}\left(u-u_{+}\right) & u \geq u_{+} \\ 0 & u_{-}<u<u_{+} \\ k_{x}\left(u-u_{-}\right) & u \leq u_{-}\end{cases}
$$



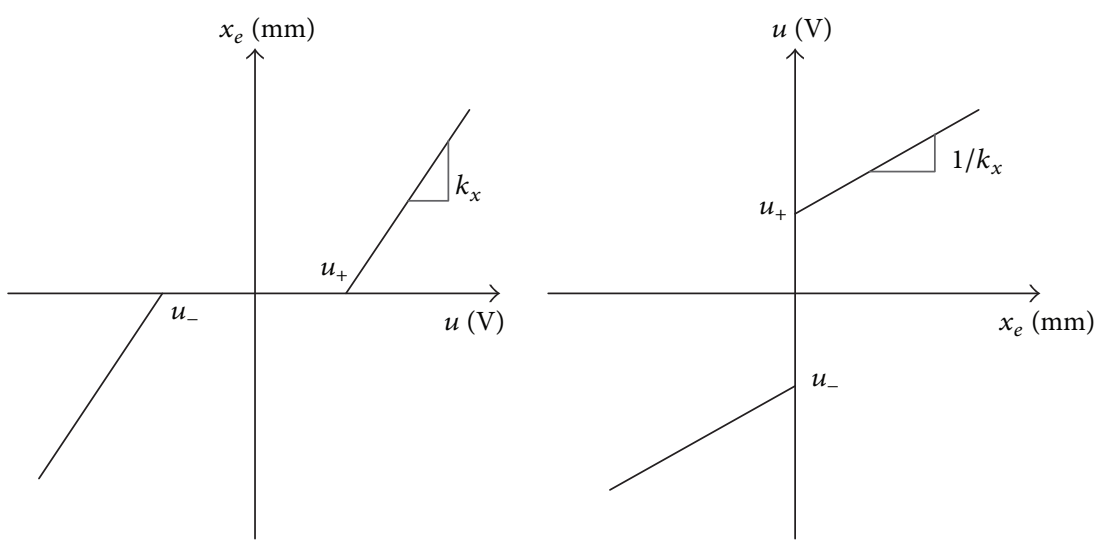

FIgURE 3: Typical deadzone mathematic model.

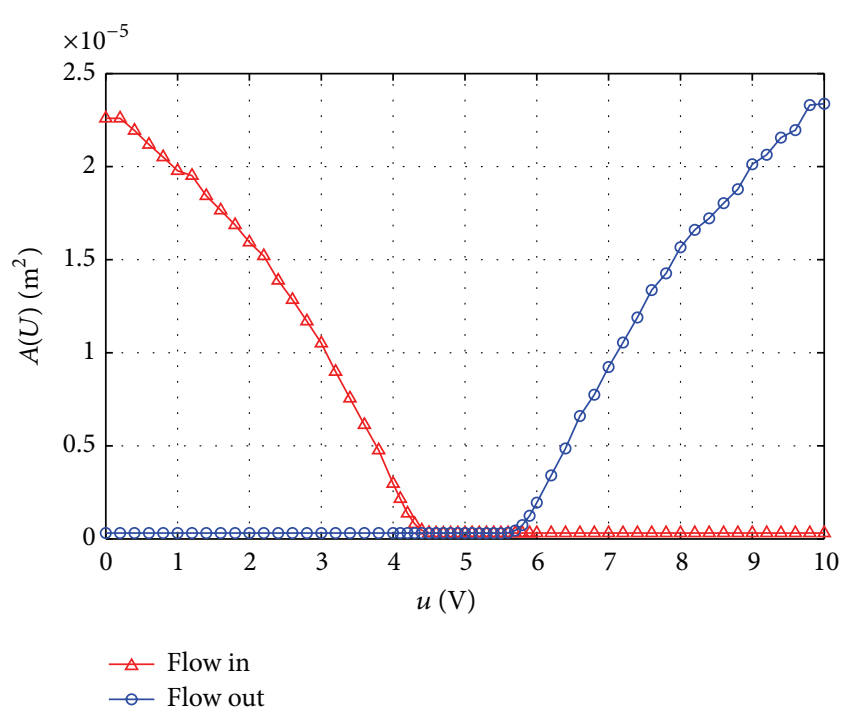

FIGURE 4: FESTO MPYE $1 / 4^{\prime}$ valve model and its deadzone property.

where $x_{e}$ is the actual open length of the proportional valve. The inverse deadzone calculation is given as follows:

$$
u=D^{-1}\left(x_{e}\right)= \begin{cases}\frac{x_{e}}{k_{x}}+\widehat{u}_{+} & x_{e}>0 \\ \frac{x_{e}}{k_{x}}+\widehat{u}_{-} & x_{e}<0\end{cases}
$$

where $\widehat{u}_{+}$and $\widehat{u}_{-}$are the estimated deadzone boundary points. Then, the mass flow $q_{m}$ can be calculated with the following equations:

$$
q_{m}=x_{e} g\left(p_{u}, p_{d}, T_{s}\right)
$$

$$
\begin{aligned}
& g\left(p_{u}, p_{d}, T_{s}\right)= \begin{cases}\frac{D C_{d} C_{a} P_{u}}{\sqrt{T_{u}}} & \frac{P_{d}}{P_{u}} \leq p_{r} \\
\frac{D C_{d} C_{a} C_{b} P_{u}}{\sqrt{T_{u}}} & p_{r}<\frac{P_{d}}{P_{u}}<\lambda \\
\frac{D C_{d} C_{a} C_{c} P_{u}}{\sqrt{T_{u}}} & \lambda \leq \frac{P_{d}}{P_{u}} \leq 1\end{cases} \\
& C_{a}=\sqrt{\left(\frac{\gamma}{R}\right)\left(\frac{2}{(\gamma+1)}\right)^{(\gamma+1) /(\gamma-1)}} \\
& C_{b}=\sqrt{1-\frac{\left(P_{d} / P_{u}-p_{r}\right)^{2}}{\left(1-p_{r}\right)^{2}}} \\
& C_{c}=\frac{\left(P_{d} / P_{u}-p_{r}\right)}{(1-\lambda)} \sqrt{1-\left[\frac{\left(\lambda-p_{r}\right)}{\left(1-p_{r}\right)}\right]^{2}},
\end{aligned}
$$

where $P_{d}$ and $P_{u}$ are the downstream and upstream pressures, respectively. $p_{r}$ is the critical pressure ratio, and $\lambda$ is the linear flow ratio $[28,29]$.

\section{Controller Design and Estimation}

Based on the dynamic model of the 3-RPS pneumatic system, the unknown parameter matrices can be created as the following matrix definitions:

$$
\begin{aligned}
& \boldsymbol{\varphi}_{a}^{T} \\
& =\left[\begin{array}{ccccccccc}
\dot{x}_{1} & S_{f 1}\left(\dot{x}_{1}\right) & -1 & 0 & 0 & 0 & 0 & 0 & 0 \\
0 & 0 & 0 & \dot{x}_{2} & S_{f 2}\left(\dot{x}_{2}\right) & -1 & 0 & 0 & 0 \\
0 & 0 & 0 & 0 & 0 & 0 & \dot{x}_{3} & S_{f 3}\left(\dot{x}_{3}\right) & -1
\end{array}\right] \\
& \boldsymbol{\theta}_{a}=\left[\theta_{1}, \theta_{2}, \theta_{3}\right]^{T} \\
& \boldsymbol{\theta}_{i}=\left[b_{i}, A_{f i},-F_{L i}+f_{n i}\right]^{T} i=1,2,3 .
\end{aligned}
$$


Then, the dynamic model in (4) of the system can be simplified as

$$
\mathbf{M}_{e} \ddot{\mathbf{x}}=\bar{A}_{a} \mathbf{p}_{L}+\boldsymbol{\varphi}_{a}^{T} \boldsymbol{\theta}_{a}+\widetilde{\mathbf{f}}_{0} .
$$

For the thermal process model part in the nonlinear model of the system as a whole, the unknown parameters can be defined as follows:

$$
\begin{aligned}
& \boldsymbol{\theta}_{q a}=\left[\theta_{b 1}, \theta_{z 1}, \theta_{b 2}, \theta_{z 2}, \theta_{b 3}, \theta_{z 3}\right]^{T} \\
& =\left[d_{n 1}, d_{z a 1}, d_{n 2}, d_{z a 2}, d_{n 3}, d_{z a 3}\right]^{T} \\
& \boldsymbol{\theta}_{q b}=\left[\theta_{b 4}, \theta_{z 4}, \theta_{b 5}, \theta_{z 5}, \theta_{b 6}, \theta_{z 6}\right]^{T} \\
& =\left[d_{n 4}, d_{z b 1}, d_{n 5}, d_{z b 2}, d_{n 6}, d_{z b 3}\right]^{T} \\
& \boldsymbol{\varphi}_{q a}^{T}=\left[\begin{array}{cccccc}
-1 & \phi_{a 1} & 0 & 0 & 0 & 0 \\
0 & 0 & -1 & \phi_{a 2} & 0 & 0 \\
0 & 0 & 0 & 0 & -1 & \phi_{a 3}
\end{array}\right] \\
& \boldsymbol{\varphi}_{q b}^{T}=\left[\begin{array}{cccccc}
-1 & -\phi_{b 1} & 0 & 0 & 0 & 0 \\
0 & 0 & -1 & -\phi_{b 2} & 0 & 0 \\
0 & 0 & 0 & 0 & -1 & -\phi_{b 3}
\end{array}\right] \text {, }
\end{aligned}
$$

where $\phi_{a i}=\left(\gamma R T_{s} / H_{p} V_{a i}\right) g\left(p_{s}, p_{a i}, T_{s}\right) k_{x i}, \phi_{b i}=\left(\gamma R T_{s} /\right.$ $\left.H_{p} V_{b i}\right) g\left(p_{s}, p_{b i}, T_{s}\right) k_{x i}$.

Considering the modeling analysis above, the systems total model can be expressed as

$$
\begin{aligned}
\dot{\mathbf{x}}_{1} & =\mathbf{x}_{2} \\
\mathbf{M}_{e} \dot{\mathbf{x}}_{2} & =\bar{A}_{a} \mathbf{p}_{L}-\boldsymbol{\varphi}_{a}^{T} \boldsymbol{\theta}_{a}+\widetilde{\mathbf{f}}_{0} \\
\dot{\mathbf{x}}_{3} & =\mathbf{q}_{L a}+\mathbf{F}_{p a}-\boldsymbol{\varphi}_{q a}^{T} \boldsymbol{\theta}_{q a}+\widetilde{\mathbf{d}}_{0 a} \\
\dot{\mathbf{x}}_{4} & =\mathbf{q}_{L b}+\mathbf{F}_{p b}-\boldsymbol{\varphi}_{q b}^{T} \boldsymbol{\theta}_{q b}+\widetilde{\mathbf{d}}_{0 b} \\
\boldsymbol{\theta}_{i} & \in \mathbf{B}_{\theta_{i}} \\
\boldsymbol{\theta}_{q a, q b} & \in \mathbf{B}_{\theta_{q a, q b}} \\
\widetilde{\mathbf{f}}_{0} & \in \mathbf{B}_{\tilde{f}_{0}} \\
\widetilde{\mathbf{d}}_{0 a} & \in \mathbf{B}_{\widetilde{d}_{0 a}} \\
\widetilde{\mathbf{d}}_{0 b} & \in \mathbf{B}_{\widetilde{d}_{0 b}} .
\end{aligned}
$$

Although accurate values of $\boldsymbol{\theta}_{i}, \boldsymbol{\theta}_{q a}, \boldsymbol{\boldsymbol { \theta } _ { q b }}, \widetilde{\mathbf{f}}_{0}$, and $\widetilde{\mathbf{d}}_{0 a}, \widetilde{\mathbf{d}}_{0 b}$ are unknown, the parameters all have definite physical meanings and they should all be bounded. $\mathbf{B}_{\boldsymbol{\theta}_{i}}, \mathbf{B}_{\theta_{q a}}, \mathbf{B}_{\boldsymbol{\theta}_{q b}}, \mathbf{B}_{\tilde{f}_{0}}$, and $\mathbf{B}_{\tilde{d}_{0 a}}$, $\mathbf{B}_{\widetilde{d}_{0 b}}$ are the boundary restriction interval for the unknown parameters and the nonlinear uncertainties.

3.1. Online Parameters Estimation. According to the dynamic analysis of the 3-RPS platform, the unknown parameters are important for control performance. However, not all of them can be measured in advance, and the friction parameters may change as the temperature or load distribution varies. Also, as mentioned above, the deadzone is key to precision and cannot be set as constant. Moreover, the load features will not be the same as the last operation in an actual working situation. As noted above, given that the unknown parameters are all bounded, the least-squares method (LSM) can be used to estimate them.

Based on the concept of parameters' online estimation [11, 30], $G_{f}$ is set as a stable LTI transfer function whose order number is larger than or equal to 3 . This filter is used to obtain the status values $\mathbf{y}_{i f}$ for the parameter estimation:

$$
G_{f}=\frac{\omega_{f}^{2}}{\left(\tau_{f} s+1\right)\left(s^{2}+2 \xi \omega_{f} s+\omega_{f}^{2}\right)} .
$$

Assuming $\widetilde{\mathbf{f}}_{0}=\widetilde{\mathbf{d}}_{0}=\mathbf{0}$, multiply $G_{f}$ with each side of (12). By defining $\boldsymbol{\varphi}_{i f}^{T}=G_{f} \boldsymbol{\varphi}_{i}^{T}$, we obtain the following equations:

$$
\begin{gathered}
\mathbf{y}_{a f}=G_{f}\left[\mathbf{M}_{e} \ddot{\mathbf{x}}-\bar{A}_{a} \mathbf{p}_{L}\right]=\boldsymbol{\varphi}_{a f}^{T} \boldsymbol{\theta}_{a} \\
\mathbf{y}_{q a f}=G_{f}\left[-\dot{\mathbf{p}}_{a}+\mathbf{q}_{L a}+\mathbf{F}_{p a}\right]=\boldsymbol{\varphi}_{q a f}^{T} \boldsymbol{\theta}_{q a} \\
\mathbf{y}_{q b f}=G_{f}\left[-\dot{\mathbf{p}}_{b}+\mathbf{q}_{L b}+\mathbf{F}_{p b}\right]=\boldsymbol{\varphi}_{q b f}^{T} \boldsymbol{\theta}_{q b},
\end{gathered}
$$

which can be written as $\mathbf{y}_{i f}=\boldsymbol{\varphi}_{i f}^{T} \boldsymbol{\theta}_{i}, i=a, q a, q b$, and is in standard linear regression form.

Use $\boldsymbol{\varepsilon}_{i}$ to represent the predictive error vector:

$$
\boldsymbol{\varepsilon}_{i}=\widehat{\mathbf{y}}_{i f}-\mathbf{y}_{i f}=\boldsymbol{\varphi}_{i f}^{T} \widehat{\boldsymbol{\theta}}_{i}-\boldsymbol{\varphi}_{i f}^{T} \boldsymbol{\theta}_{i}=\boldsymbol{\varphi}_{i f}^{T} \widetilde{\boldsymbol{\theta}}_{i} .
$$

The equation above is the standard parameter estimation model. LSM could be used to obtain the estimated vector $\widehat{\boldsymbol{\theta}}_{i}$.

Moreover, noncontinuous projection mapping is used to ensure that the estimation results are bounded:

$$
\dot{\hat{\boldsymbol{\theta}}}=\operatorname{sat}_{\dot{\boldsymbol{\theta}}_{M}}\left(\operatorname{Pr}_{\hat{\theta}}(\boldsymbol{\Psi} \boldsymbol{\tau})\right) \quad \widehat{\boldsymbol{\theta}}(0) \in B_{\boldsymbol{\theta}},
$$

where $\Psi$ is the positive definite adaptive matrix and $\boldsymbol{\tau}$ is the adaptive function. $B_{\theta}$ is the known boundaries set of the unknown parameters. $\operatorname{Pr}_{\hat{\theta}}$ is the standard projection mapping. This projection mapping has been described in Goodwin's and Yao's works. sat $\dot{\theta}_{M}(\bullet)$ is the saturation function, which is used to limit the updating speed of the parameters. The saturation function is defined as

$$
\operatorname{sat}_{\dot{\boldsymbol{\theta}}_{M}}(\bullet)=s_{a} \cdot, \quad s_{a}= \begin{cases}1 & \|\bullet\| \leq \dot{\hat{\boldsymbol{\theta}}}_{M} \\ \frac{\dot{\hat{\boldsymbol{\theta}}}_{M}}{\|\bullet\|} & \|\bullet\|>\dot{\hat{\boldsymbol{\theta}}}_{M},\end{cases}
$$

where $\dot{\hat{\boldsymbol{\theta}}}_{M}$ is the maximum parameter updating speed determined in advance. Using this approach, the estimated parameters would always be inside the closed set of $B_{\boldsymbol{\theta}}$. 
The adaptive rate matrix $\Psi_{i}$ is shown in

$$
\dot{\boldsymbol{\Psi}}_{i}= \begin{cases}\alpha_{i} \Psi_{i}-\frac{\boldsymbol{\Psi}_{i} \boldsymbol{\varphi}_{i f} \boldsymbol{\varphi}_{i f}^{T} \boldsymbol{\Psi}_{i}}{1+v_{i} \boldsymbol{\varphi}_{i f}^{T} \boldsymbol{\Psi}_{i} \boldsymbol{\varphi}_{i f}} & \text { when } \lambda_{\max }\left(\boldsymbol{\Psi}_{i}(t)\right) \leq \rho_{M i},\left\|\operatorname{Pr}_{\widehat{\theta}}\left(\Psi_{i} \boldsymbol{\tau}_{i}\right)\right\| \leq \widehat{\boldsymbol{\theta}}_{M i} \\ \mathbf{0} & \text { otherwise, }\end{cases}
$$

where $\alpha_{i} \geq 0$ is the fading factor that reduces the influence caused by the old data. $v \geq 0$ is the normalized factor, $\lambda_{\text {max }}\left(\Psi_{i}(t)\right)$ is the largest eigenvalue of $\Psi_{i}(t)$, and $\rho_{M i}$ is the upper bound of $\left\|\Psi_{i}(\mathbf{t})\right\| . \boldsymbol{\tau}_{i}$ is the adaptive function, as shown in

$$
\boldsymbol{\tau}_{i}=\frac{1}{1+v_{i} \boldsymbol{\varphi}_{i f}^{T} \boldsymbol{\Psi}_{i} \boldsymbol{\varphi}_{i f}} \boldsymbol{\varphi}_{i f} \boldsymbol{\varepsilon}_{i}
$$

3.2. Controller Structure. Based on back-stepping concepts, there are two steps in designing the controller that finally export the calculated control values for the proportional valves. The first step is considered at the level of pressure. $\mathbf{e}_{s}$ is defined as a vector that is similar to the sliding model surface vector. $\mathbf{K}_{x}$ is the constant matrix chosen as a proportional feedback of the tracking error vector e:

$$
\begin{aligned}
& \mathbf{e}_{s}=\dot{\mathbf{e}}+\mathbf{K}_{x} \mathbf{e} \\
& \dot{\mathbf{e}}_{s}=\ddot{\mathbf{e}}+\mathbf{K}_{x}\left(\dot{\mathbf{x}}-\dot{\mathbf{x}}_{d}\right) .
\end{aligned}
$$

$\mathbf{p}_{L}$ is used as the first-level virtual input and $\mathbf{e}_{p}=\mathbf{p}_{L}-$ $\mathbf{p}_{L d}$ is used to describe the error between the desired pressure vector $\mathbf{p}_{L d}$ and the measured pressure vector $\mathbf{p}_{L}$ :

$$
\mathbf{p}_{L d}=\mathbf{p}_{L d a 1}+\mathbf{p}_{L d a 2}+\mathbf{p}_{L d s 1}+\mathbf{p}_{L d s 2}
$$

where $\mathbf{p}_{L d a 1}$ is the model compensation part. $\mathbf{p}_{L d s 1}$ is chosen as the proportional feedback to stabilize the ideal modeled dynamic system:

$$
\begin{aligned}
& \mathbf{p}_{L d a 1}=\frac{1}{\bar{A}_{a}}\left[-\boldsymbol{\varphi}_{a}^{T} \widehat{\boldsymbol{\theta}}_{a}+\mathbf{M}_{e} \ddot{\mathbf{x}}_{d}-\mathbf{M}_{e} \mathbf{K}_{x} \mathbf{e}\right] \\
& \mathbf{p}_{L d s 1}=-\frac{1}{\bar{A}_{a}} \mathbf{K}_{p} \mathbf{e}_{s},
\end{aligned}
$$

where $\mathbf{K}_{p}$ is a positive definite diagonal matrix. $\mathbf{p}_{L d a 2}$ is the fast compensation part. $\mathbf{p}_{L d s 2}$ is the robust feedback part that inhibits the influence caused by parameter estimation error and uncertain nonlinearity. This part can be defined as

$$
\begin{aligned}
\mathbf{d}_{c 1}+\mathbf{R}_{1}(t) & =-\boldsymbol{\varphi}_{a}^{T} \widetilde{\boldsymbol{\theta}}_{a}+\widetilde{\mathbf{f}}_{0} \\
\mathbf{p}_{L d a 2} & =-\frac{1}{\bar{A}_{a}} \widehat{\mathbf{d}}_{c 1},
\end{aligned}
$$

where $\mathbf{d}_{c 1}$ is the low-frequency part of the estimation error and $\mathbf{R}_{1}(t)$ is the high-frequency part. $\mathbf{d}_{c 1}$ can be estimated readily and is used to calculate $\mathbf{p}_{L d a 2} \cdot \mathbf{p}_{L d s 2}$ is defined by the equation below:

$$
\begin{aligned}
& \mathbf{p}_{L d s 2}=-\frac{1}{\bar{A}_{a}} \frac{H_{1}^{2}(t)}{4 \eta_{1}} \mathbf{e}_{s} \\
& H_{1}(t) \geq d_{c 1 M}+\left\|\boldsymbol{\theta}_{M a}\right\|\left\|\boldsymbol{\varphi}_{a}\right\|+f_{\max },
\end{aligned}
$$

where $d_{c 1 M}$ is the upper bound of $\left\|\mathbf{d}_{c 1}\right\|$ and $\boldsymbol{\theta}_{M a}=\boldsymbol{\theta}_{\max a}-$ $\boldsymbol{\theta}_{\min a}$.

The purpose of the second-level controller design is to make $\mathbf{e}_{p}=\mathbf{p}_{L}-\mathbf{p}_{L d}$ converge to zero. Then, the derivation of $\mathbf{e}_{p}$ is given by

$$
\dot{\mathbf{e}}_{p}=\mathbf{q}_{L}+\mathbf{F}_{p}-\boldsymbol{\varphi}_{q a}^{T} \boldsymbol{\theta}_{q a}+\boldsymbol{\varphi}_{q b}^{T} \boldsymbol{\theta}_{q b}+\tilde{\mathbf{d}}_{0 a}-\tilde{\mathbf{d}}_{0 b}-\dot{\mathbf{p}}_{L d}
$$

where

$$
\begin{aligned}
& q_{L i} \\
& =\left\{\begin{array}{l}
\frac{\gamma R T_{s}}{H_{p} V_{a i}} g\left(p_{s}, p_{a i}, T_{s}\right) x_{e i}+\frac{\gamma R T_{b}}{H_{p} V_{b i}} g\left(p_{b}, p_{0}, T_{b}\right) x_{e i} \quad x_{e i} \geq 0 \\
\frac{\gamma R T_{a}}{H_{p} V_{a i}} g\left(p_{a}, p_{0}, T_{a}\right) x_{e i}+\frac{\gamma R T_{s}}{H_{p} V_{b i}} g\left(p_{s}, p_{b}, T_{s}\right) x_{e i} \quad x_{e i}<0,
\end{array}\right. \\
& F_{p i}=-\frac{\gamma A_{i a}}{V_{i a}} \dot{x}_{1 i} p_{i a}-\frac{\gamma A_{i b}}{V_{i b}} \dot{x}_{1 i} p_{i b}+\frac{\gamma-1}{H_{p} V_{i a}} \dot{Q}_{i a}-\frac{\gamma-1}{H_{p} V_{i b}} \dot{Q}_{i b} .
\end{aligned}
$$

The error of the deadzone estimation can be calculated using the following equation:

$$
\begin{aligned}
\Delta_{D i}(t)= & x_{e i}-x_{e d i}=D\left[D^{-1}\left(x_{e d i}\right)\right]-x_{e d i} \\
= & \begin{cases}k_{x i} \widetilde{\theta}_{+i} & u_{i} \geq u_{+i} \\
-x_{e d i} & u_{+i}>u_{i}>u_{-i} \\
k_{x i} \widetilde{\theta}_{-i} & u_{-i}>u_{i},\end{cases}
\end{aligned}
$$

where $i=1,2,3$ and $x_{e d i}$ is the desired open length of each proportional valve.

Equation (26) indicates that this step is considered in the level of flow rate. Because the flow rate is determined by the control voltage directly, the result of this step can be used in the calculation of the control value vector $\mathbf{u}_{c}$ :

$$
\mathbf{q}_{L}=\mathbf{q}_{L d a 1}+\mathbf{q}_{L d a 2}+\mathbf{q}_{L d s 1}+\mathbf{q}_{L d s 2}
$$

Similar to the first step of controller design of $\mathbf{p}_{L}, \mathbf{q}_{L d a 1}$ is the model compensation part. $\mathbf{q}_{L d s 1}$ is chosen as the 


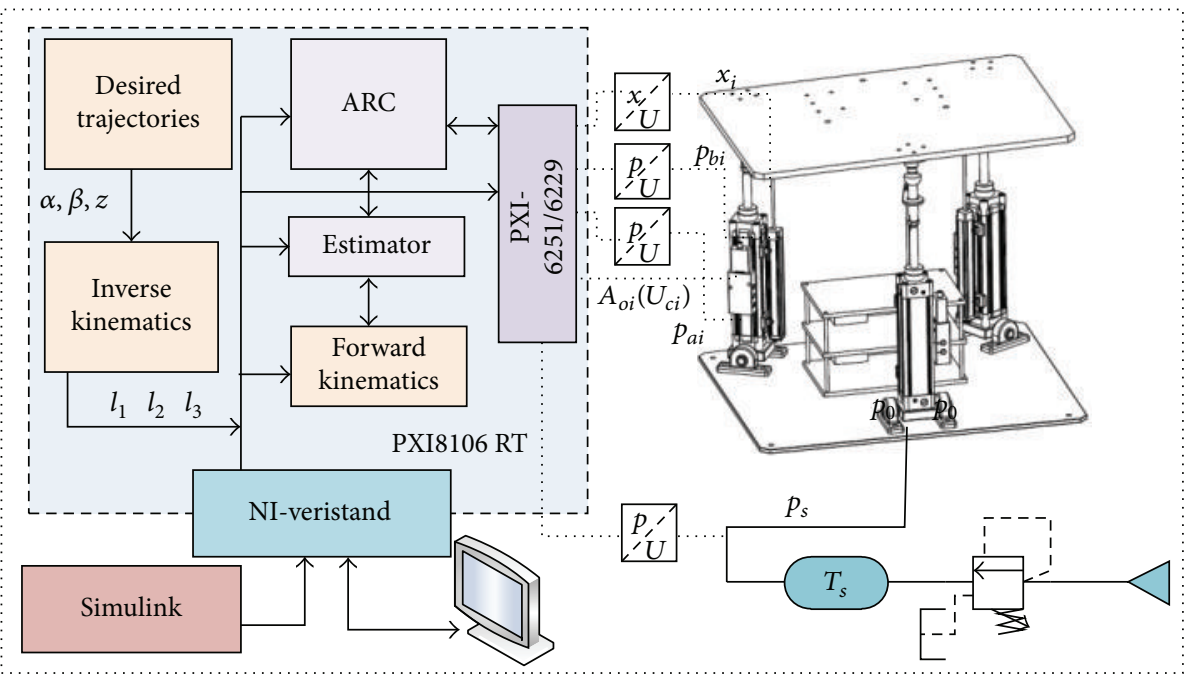

FIGURE 5: Control system and simplified signal interfaces configurations diagram.

proportional feedback to stabilize the ideal modeled dynamic system:

$$
\begin{aligned}
& \mathbf{q}_{L d a 1}=-\bar{A}_{a} \mathbf{e}_{s}-\mathbf{F}_{p}-\boldsymbol{\varphi}_{b}^{T} \widehat{\boldsymbol{\theta}}_{b}+\dot{\mathbf{p}}_{L d} \\
& \mathbf{q}_{L d s 1}=-\mathbf{K}_{q} \mathbf{e}_{p},
\end{aligned}
$$

where $\mathbf{K}_{q}$ is a positive definite diagonal matrix. $\mathbf{q}_{L d a 2}$ is the fast compensation part. $\mathbf{q}_{L d s 2}$ is the robust feedback part that inhibits the influence caused by parameter estimation error and uncertain nonlinearity. This part can be defined as

$$
\begin{aligned}
\mathbf{d}_{c 2}+\mathbf{R}_{2}(\mathbf{t}) & =\mathbf{q}_{L g} \Delta_{D}-\boldsymbol{\varphi}_{b}^{T} \widetilde{\boldsymbol{\theta}}_{b}+\widetilde{\mathbf{d}}_{0} \\
\mathbf{q}_{L d a 2} & =-\widehat{\mathbf{d}}_{c 2},
\end{aligned}
$$

where $\mathbf{q}_{L g}=\left[q_{L 1} / x_{e 1}, q_{L 2} / x_{e 2}, q_{L 3} / x_{e 3}\right]^{T}, \mathbf{d}_{c 2}$ is the lowfrequency part of the estimation error, and $\mathbf{R}_{2}(t)$ is the highfrequency part. $\mathbf{d}_{c 2}$ can be estimated readily and used to calculate $\mathbf{q}_{L d a 2} \cdot \mathbf{q}_{L d s 2}$ is defined by the equation below:

$$
\begin{aligned}
\mathbf{q}_{L d s 2}= & -\frac{H_{2}^{2}(t)}{4 \eta_{2}} \mathbf{e}_{p} \\
H_{2}(t) \geq & \left\|\mathbf{M}_{e}^{-1} \frac{\partial \dot{\mathbf{p}}_{L d}}{\partial \dot{\mathbf{x}}}\right\|\left(\left\|\boldsymbol{\theta}_{M a}\right\|\left\|\boldsymbol{\varphi}_{a}\right\|+f_{\max }\right)+\mathbf{d}_{c 2 M} \\
& +\left\|\boldsymbol{\theta}_{M b}\right\|\left\|\boldsymbol{\varphi}_{b}\right\|+\mathbf{d}_{\max }+\left\|\mathbf{q}_{L g}\right\|\left\|\Delta_{D}\right\|,
\end{aligned}
$$

where $d_{c 2 M}$ is the upper bound of $\left\|\mathbf{d}_{c 2}\right\|$ and $\boldsymbol{\theta}_{M b}=\boldsymbol{\theta}_{\max b}-$ $\boldsymbol{\theta}_{\min b}$.

Finally, when $\mathbf{q}_{L d}$ is calculated, the inverse processing of the flow rate calculation is applied according to

$$
x_{e d i}=q_{L g i}^{-1} q_{L d i} .
$$

Determined by the valve testing experiment results shown in Figure 4, the relationship between control voltage and valve's open length can be expressed as

$$
\begin{aligned}
& x_{\text {edi }}=J\left(u_{c i}\right) \\
& = \begin{cases}J_{a}\left(u_{c i}\right)=K_{A} u_{c i}^{2}+K_{B} u_{c i}+K_{C} & u_{c i} \geq \theta_{z i} \\
J_{b}\left(u_{c i}\right)=0 & \theta_{z(i+3)}<u_{c i}<\theta_{z i} \\
J_{c}\left(u_{c i}\right)=K_{D} u_{c i}^{2}+K_{E} u_{c i}+K_{F} & u_{c i} \geq \theta_{z(i+3)},\end{cases}
\end{aligned}
$$

where $u_{c i}$ is real output voltage, $x_{e d i}$ is the desired open length of each proportional valve, and $K_{A}-K_{F}$ are constants to describe the flow rate curve. Then, the real output voltage vector $\mathbf{u}_{c}=\left[u_{c 1}, u_{c 2}, u_{c 3}\right]^{T}$ is given by

$$
u_{c i}=J^{-1}\left(x_{e d i}\right)= \begin{cases}J_{a}^{-1}\left(x_{e d i}\right)+\widehat{\theta}_{z i} & x_{e d i}>0 \\ J_{c}^{-1}\left(x_{e d i}\right)+\widehat{\theta}_{z(i+3)} & x_{e d i}<0 .\end{cases}
$$

3.3. Controller Simulation. The NI-PXI system was used as a physical controller. The controller consisted of the estimation part, core adaptive robust controller part, and the kinematics calculation parts. The data interactive environment from the Simulink model was used to embed software. The control system structure and the interfaces configurations of the actual 3-RPS platform are shown in Figure 5. Pressure and position sensors were attached to the plant. Before applying the algorithms to a physical plant, a simulation process was performed to verify the effectiveness of the online deadzone estimation. Sine waves were set as the desired reference curves for the three cylinders, as follows: $x d(i)=$ $0.09 \sin (1.4 t+2 \pi(i-1) / 3), \quad i=1,2,3$.

To provide a clear comparison between different simulation and experimental results, the controller began working $2 \mathrm{~s}$ after the RUN command was ordered. Data logging started $4 \mathrm{~s}$ from the beginning of the control experiment (i.e., the time origin point in the following error and data analysis 
TABLE 1: Constant values used in the model.

\begin{tabular}{|c|c|c|}
\hline Symbols & Descriptions & Value \\
\hline$A_{a}$ & Chamber A area & $31.2 \times 10^{-4} \mathrm{~m}^{2}$ \\
\hline$A_{b}$ & Chamber B area & $28.03 \times 10^{-4} \mathrm{~m}^{2}$ \\
\hline$R$ & Air constant & $287 \mathrm{Nm} /(\mathrm{Kg} \cdot \mathrm{K})$ \\
\hline$T_{s}$ & Air source temperature & $295 \mathrm{~K}$ \\
\hline$L$ & Cylinder length & $0.2 \mathrm{~m}$ \\
\hline$\gamma_{a}$ & Heat capacity ratio & 1.4 \\
\hline$\tau_{f}, \omega_{f}, \xi_{f}$ & Filter parameters & $1,50,0.1$ \\
\hline$V_{o} i$ & Dead volume & $0.2 \mathrm{~L}$ \\
\hline$h$ & Heat transfer constant & $50 \mathrm{~W} /\left(\mathrm{m}^{2} \mathrm{~K}\right)$ \\
\hline$D$ & Cylinder diameter & $0.063 \mathrm{~m}$ \\
\hline$\alpha_{i}$ & Fading factor & 0.1 \\
\hline$v_{i}$ & Normalization factor & 0.1 \\
\hline$H_{1}(t), \eta_{1}$ & $\mathbf{p}_{\mathrm{Lds} 2}$ constant & 80,4 \\
\hline$H_{2}(t), \eta_{2}$ & $\mathbf{q}_{\mathrm{Lds} 2}$ constant & 200,10 \\
\hline $\mathbf{K}_{x}$ & Error vector feedback & $\operatorname{diag}(45,45,45)$ \\
\hline $\mathbf{K}_{p}$ & $\mathbf{p}_{\text {Lds1 }}$ feedback & $\operatorname{diag}(20,20,20)$ \\
\hline $\mathbf{K}_{q}$ & $\mathbf{q}_{\mathrm{Lds} 1}$ feedback & $\operatorname{diag}(60,60,60)$ \\
\hline$\Psi_{a i}(0)$ & Initialization & $\operatorname{diag}(100,100, \ldots, 100)_{9 \times 9}$ \\
\hline$\Psi_{q a i}(0)$ & Initialization & $\operatorname{diag}(100,100, \ldots, 100)_{6 \times 6}$ \\
\hline$\Psi_{q b i}(0)$ & Initialization & $\operatorname{diag}(100,100, \ldots, 100)_{6 \times 6}$ \\
\hline$d_{c 1 M}$ & Boundary of fast compensation & 10 \\
\hline$d_{c 2 M}$ & Boundary of fast compensation & 10 \\
\hline$\rho_{M 1}$ & The upper bound of $\left\|\Psi_{a i}(\mathbf{t})\right\|$ & 1000 \\
\hline$\rho_{M 2}$ & The upper bound of $\left\|\Psi_{a q i}(\mathbf{t})\right\|$ & 100 \\
\hline$\rho_{M 3}$ & The upper bound of $\left\|\Psi_{b q i}(\mathbf{t})\right\|$ & 100 \\
\hline$\dot{\boldsymbol{\theta}}_{M a}$ & Max estimation speed vector & {$[10,10, \ldots, 10]_{1 \times 9}^{T}$} \\
\hline$\dot{\boldsymbol{\theta}}_{M q a}$ & Max estimation speed vector & {$[10,10, \ldots, 10]_{1 \times 6}^{T}$} \\
\hline$\dot{\boldsymbol{\theta}}_{M q b}$ & Max estimation speed vector & {$[10,10, \ldots, 10]_{1 \times 6}^{T}$} \\
\hline
\end{tabular}

figures are chosen as the start time of data logging). With this method, data having large values, occurring when the system powers up and following a step control signal, are ignored; this reduces the $y$-axis range and makes it easier to analyze the continuous curve control performance, especially the tracking error properties. Thus, in all of the experimental results that follow, the origin points of time correspond to the beginning of data logging, not the controller starting points.

Using the configurations shown in Table 1, a model of the 3-RPS pneumatic platform was built in Simulink. The unknown parameters were initialized as shown in Table 2. The desired trajectory is a sine wave on each axis. The controller ran about $50 \mathrm{~s}$ in each experiment. The posture tracking performance is shown in Figure 6. This simulation confirmed the effectiveness of the online parameters estimator and the performance of the ARC controller.

Figures 6 and 7 show that the tracking error for the positions of all three cylinders became smaller as the estimated deadzone voltages converged to the actual numbers. The deadzone properties $\mathbf{Z}_{\text {simu }}$ were set as $(0.53,-0.72)(V)$, $(0.72,-0.53)(V)$, and $(0.4,-0.8)(V)$ for valves $1-3$, respectively, and the initial estimation values for the deadzone were chosen as $(0.45,-0.45)(V)$ for all, as shown in Figure 7 and Table 2. The results demonstrate the robustness of the deadzone estimation in that despite an initial difference $>50 \%$ compared with the real numbers, the estimated results were still bounded and converged.

\section{Experiment Results}

First, the experiments were executed under a situation without deadzone online estimation or compensation. Three situations were considered in the experiments: (1.1) no deadzone online estimation, but the friction and load parts and the deadzone settings in the controller model are different from the actual values, (1.2) no parameter online estimation and the deadzone settings are also not close to the actual figures, and (1.3) parameter online estimation for friction and load, and the deadzone settings are close to the real numbers. For each situation, the desired curve in joint space is a sine wave. The tracking errors and control voltages $u_{c}(i)$ were considered in the analysis.

In Experiment 1.1, with no deadzone online estimation, the matrix $\mathbf{Z}_{e x 1}$ was set as $[(0.7,-0.6),(0.6,-0.6),(0.5$, 
TABLE 2: Initial values for estimation.

\begin{tabular}{lcc}
\hline Symbols & Descriptions & Value \\
\hline$\widehat{\boldsymbol{\theta}}_{1}(0)$ & The initial values of vector $\boldsymbol{\theta}_{1}$ in $\boldsymbol{\theta}_{a}$ & {$\left[225,20,-9.8 m_{1}\right]^{T}$} \\
$\widehat{\boldsymbol{\theta}}_{2}(0)$ & The initial values of vector $\boldsymbol{\theta}_{2}$ in $\boldsymbol{\theta}_{a}$ & {$\left[225,20,-9.8 m_{2}\right]^{T}$} \\
$\widehat{\boldsymbol{\theta}}_{3}(0)$ & The initial values of vector $\boldsymbol{\theta}_{3}$ in $\boldsymbol{\theta}_{a}$ & {$\left[225,20,-9.8 m_{3}\right]^{T}$} \\
$\boldsymbol{\theta}_{i_{\max }}, \boldsymbol{\theta}_{i \min }$ & Boundaries of estimation vectors & {$[500,150,200]^{T},[0,0,-500]^{T}$} \\
$\widehat{\boldsymbol{\theta}}_{q a}(0)$ & The initial values of vector $\boldsymbol{\theta}_{q a}$ & {$[0,0.45,0,0.45,0,0.45]^{T}$} \\
$\hat{\boldsymbol{\theta}}_{q b}(0)$ & The initial values of vector $\boldsymbol{\theta}_{q b}$ & {$[0,-0.45,0,-0.45,0,-0.45]^{T}$} \\
$\boldsymbol{\theta}_{q i_{\max }}, \boldsymbol{\theta}_{q b_{\min }}$ & Boundaries of estimation vectors & {$[10,1,10,1,10,1]^{T},[-10,-1,-10,-1,-10,-1]^{T}$} \\
\hline
\end{tabular}
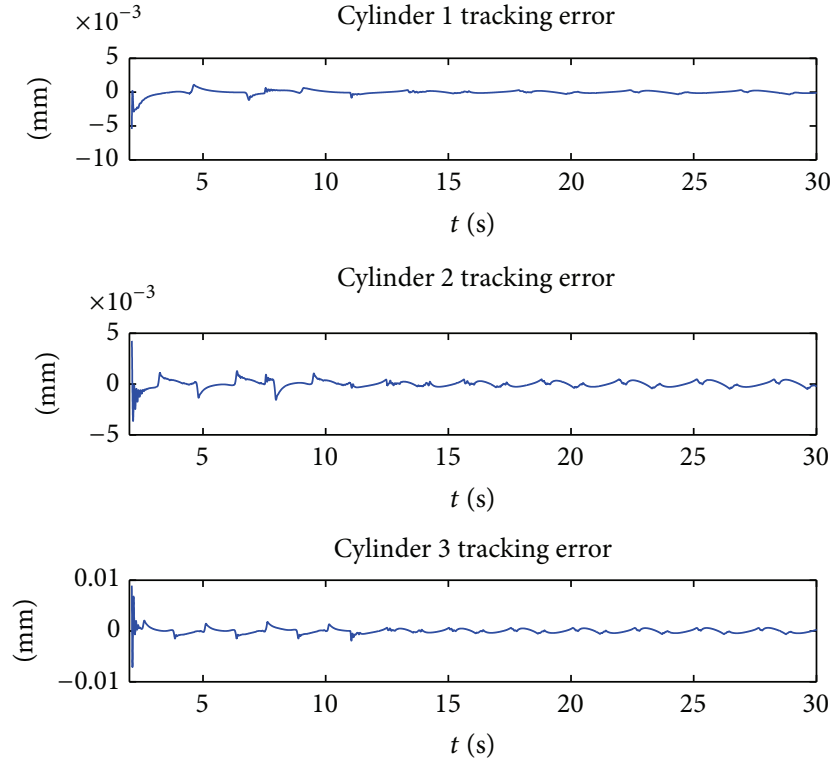

FiguRE 6: Tracking error simulation results.

$-0.6)](V)$ while the actual numbers that were determined by testing equipment in our lab were $\mathbf{Z}_{r e}=[(0.73,-0.7)$; $(0.68,-0.61) ;(0.65,-0.59)](V)$. Figure 8 demonstrates that, due to the differences in the deadzone parameters, vibration appears while controlling these cylinders and the tracking error becomes larger. The abrupt changes in the proportional valves outputs are the reason for the buffeting characteristics that occurred in Experiment 1.1, as shown in Figure 8. Moreover, it is easy to see that the heavy vibration makes the estimator for the other parameters not work properly because the tracking error becomes larger as the experiment continues.

In the situation in Experiment 1.2, the vibration became much larger, because there was no parameter estimation. Thus, the modelling error was larger than in Experiment 1.1, which will definitely influence the performance. The control outputs switching between two different working zones had a significant effect, as shown in Figure 9. Thus, the results from this experiment indicated that parameter estimation is effective and necessary in the controller design.

According to the former analysis, setting the deadzone values close to the measured characteristics improves the performance of the trajectory tracking, as shown in Figure 10; additionally, the vibration disappears, while $\mathbf{Z}_{\text {ex3 }}=[(0.7,-0.7),(0.7,-0.6),(0.65,-0.6)](V)$. However, as noted in the Introduction, each proportional valve has a unique deadzone property, even those from the same production batch. It is impossible to determine all of them before applying them in a large development situation. The algorithms with deadzone online estimation, described in Section 3.2, were tested in the following experiments.

Figure 11 presents the tracking performance in joint space with the deadzone online estimation approaches applied, which is referred to as Experiment 2.1. The left part of Figure 11 shows the tracking error of each cylinder and the right shows the control output voltage. From the left figure, it is clear that the error is becoming smaller as the control algorithm is executing. From the right figure, a quick switching phenomenon exists at the beginning period. After the estimator in the controller had worked for about $15 \mathrm{~s}$, the control output curve became smoother and the time for the switching process decreased significantly.

Figure 12 provides a workspace analysis of the tracking error results and the tracking trajectories. On each axis, it is clear that the error is also becoming smaller as the estimated parameters converge to their actual values, especially the deadzone parts. For the trajectory figure, because the control accuracy is about $1-2 \%$, it is difficult to tell the difference between the measured and desired curves.

The parameter estimation results are shown in Figure 13 for Experiment 2.1. The original deadzone values were all set as $(0.6,-0.6)(V)$ and the estimated values for the three different valves attached to different cylinders moving to the actual numbers. Approximately 10-15 s was required to reach satisfactory zones in the estimation, as shown in Figure 11.

For a more universal working situation, a set of compound workspace desired trajectories was applied in Experiment 2.2. Similarly, the error and tracking analysis are shown in Figure 14. The deadzone estimation results are shown in Figure 15. Compared with the sinewave working status in Experiment 2.1, there was no significant difference in the tracking error or deadzone estimation results, meaning that the robustness of this algorithm is acceptable. Consider

$$
\begin{aligned}
e_{F} & =\left.\max \{|e|\}\right|_{T_{f}-10 \leq t \leq T_{f}} \\
\|e\|_{\mathrm{rms}} & =\sqrt{\frac{1}{10} \int_{T_{f}-10}^{T_{f}} e^{2} d t}
\end{aligned}
$$



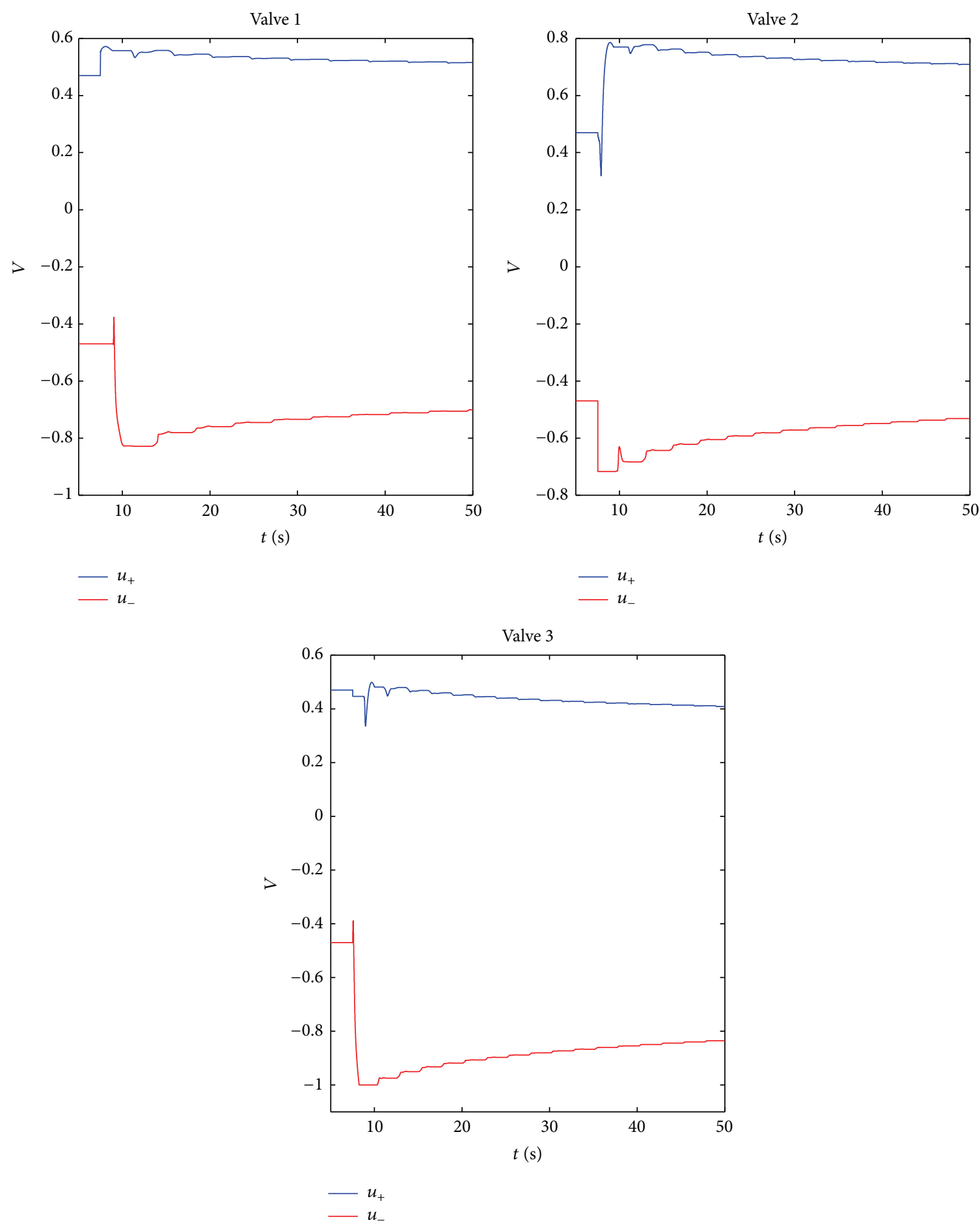

FIgURE 7: Deadzone estimation simulation results.

To summarize Experiments 1.1-1.3 and Experiments 2.12.2, error analysis must be performed to determine the effectiveness of the different control algorithms and system configurations. To quantify the performance, the data for the last $10 \mathrm{~s}$ were used to calculate the error indices. The indices include the maximum tracking error $e_{F}$ and root mean square error $\|e\|_{\mathrm{rms}}$, shown in (37). Relative error $\|e\|_{\mathrm{rms}} / A_{m}$ is calculated as a general effectiveness evaluation criterion for the controller, where $A_{m}$ is the amplitude of the trajectory. The error analyzing results are shown in Table 3 for joint space and Table 4 for workspace.

\section{Conclusion}

In this paper, we proposed an ARC controller with a deadzone compensation method. The controller is effective and stable 

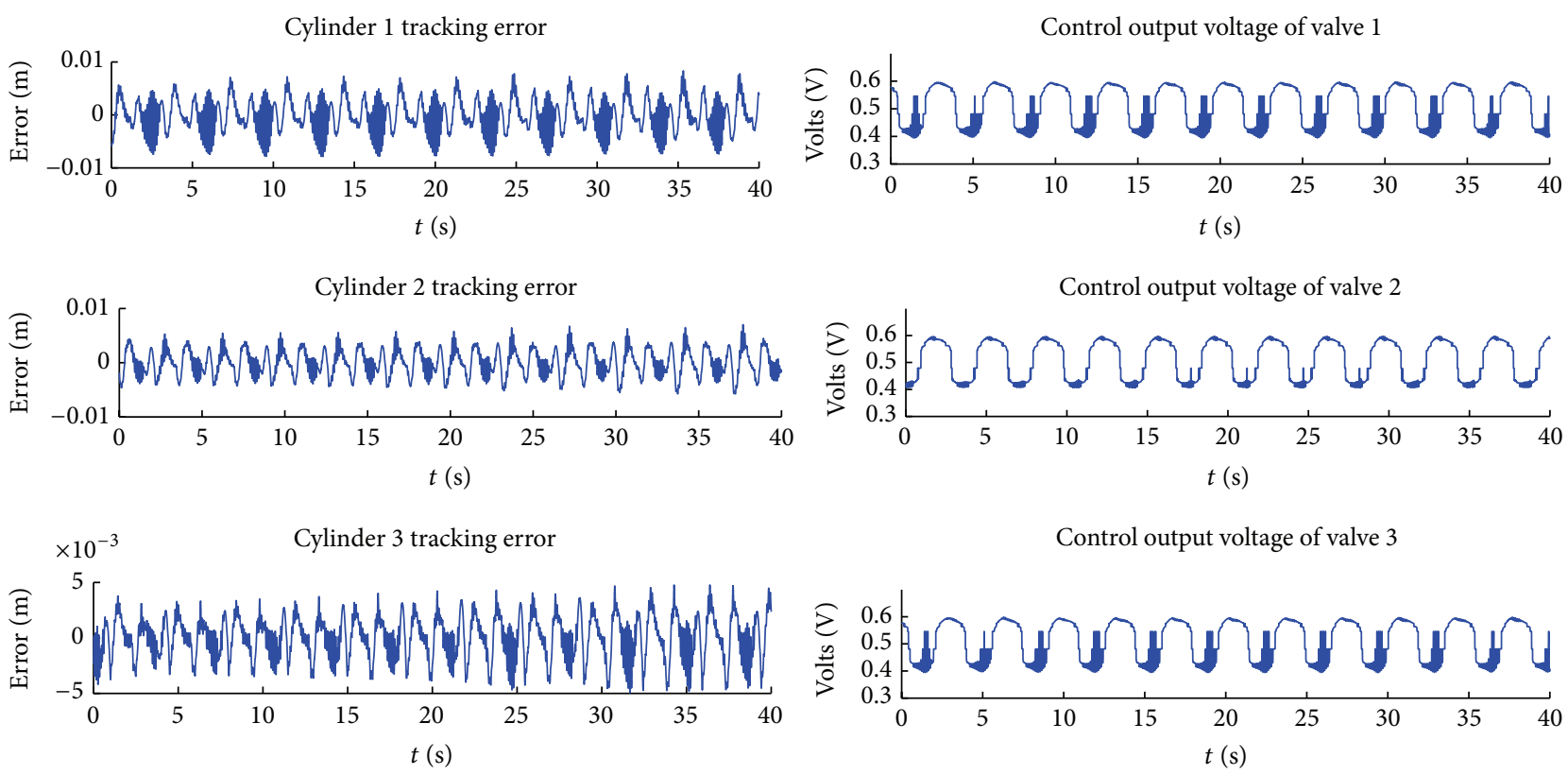

Control output voltage of valve 3

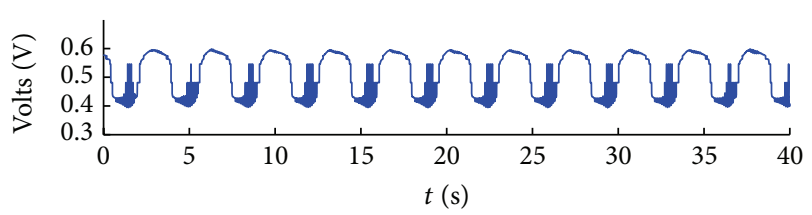

FIGURE 8: Joint space tracking error and control voltages for Experiment 1.1.
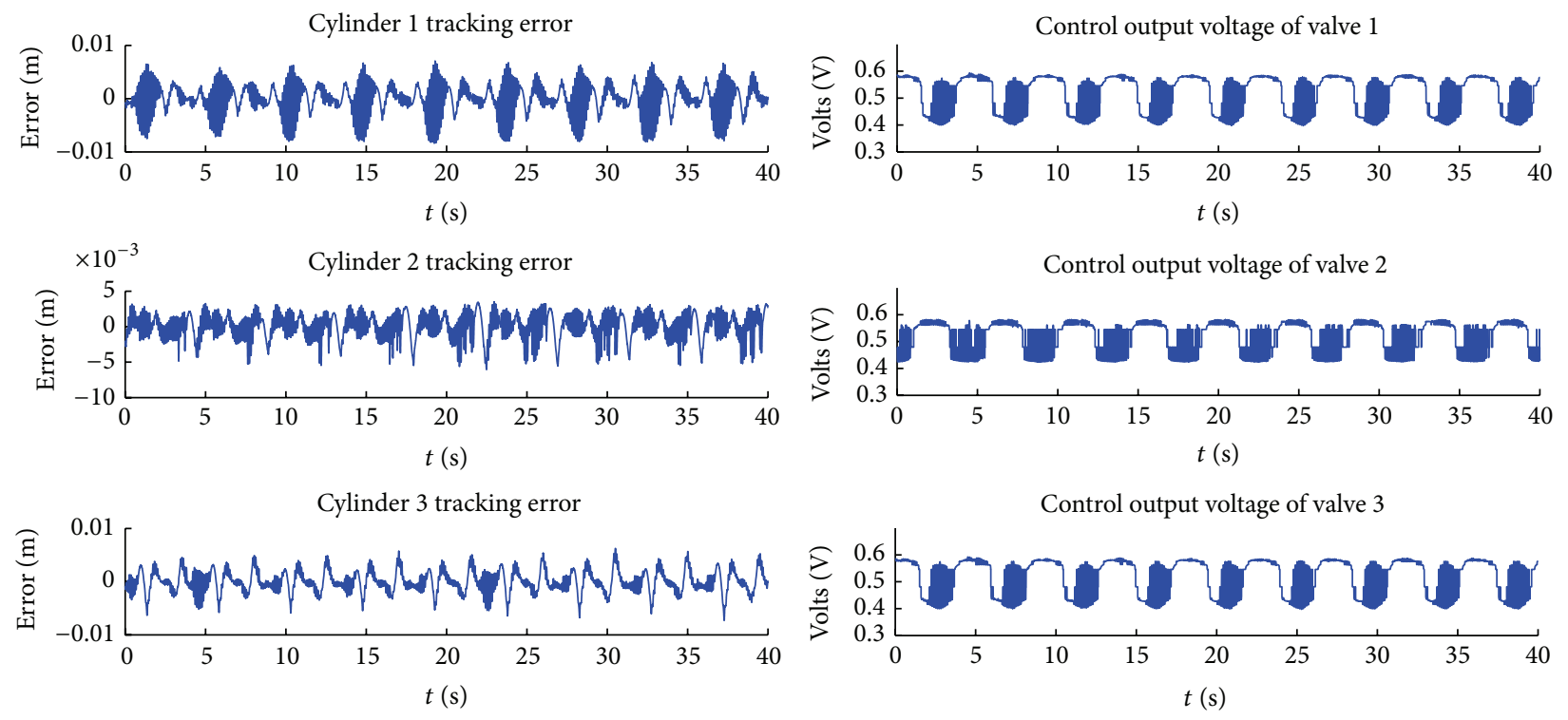

FIGURE 9: Joint space tracking error and control voltages for Experiment 1.2.

while controlling the posture of a pneumatic 3-RPS platform. The stability proof is shown in the Appendix.

Parameter uncertainty and nonlinear features can be estimated and compensated for by online estimation methods and the robust parts of the controller. Moreover, the deadzone feature estimation makes the algorithm more adaptable and strengthens the controllers universality. The experimental results indicated that the unknown parameters were indeed bounded and converged to the actual values.

In future work, to achieve higher precision for the posture, new friction models should be considered in the controller design, such as the LuGre model, which has been shown to be an effective model for the friction between the piston and cylinder. Moreover, in this controller structure, for a specific cylinder among the three, the tracking errors of the other two are not considered as feedback information. Recent research showed that it will improve the tracking performance in the workspace if all the errors for each piston are adopted into the controller design, which is referred to as cross-coupling control [31, 32]. This method may be integrated into the algorithm design to achieve better posture control results and will enable further multiplatform synchronization control research. 

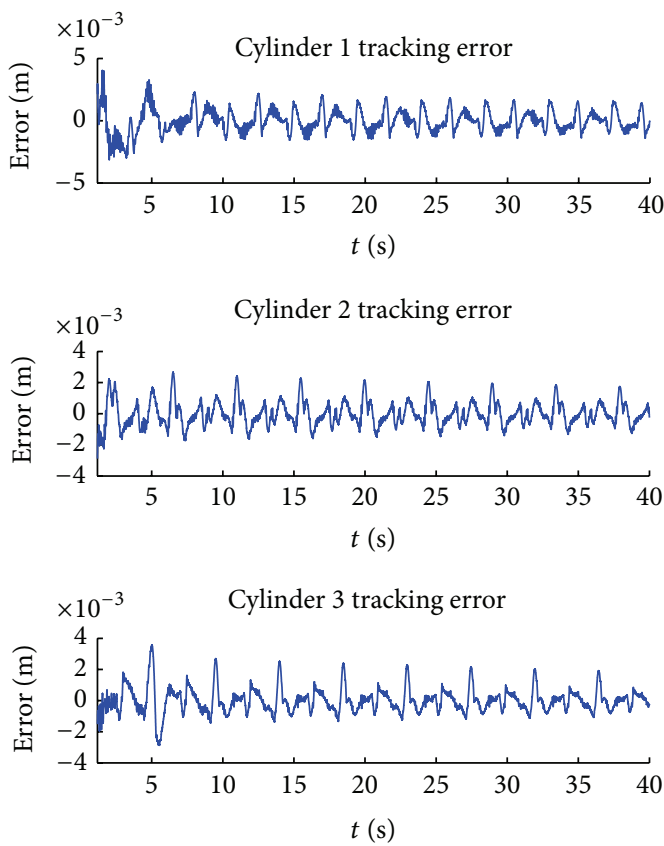

Control output voltage of valve 1

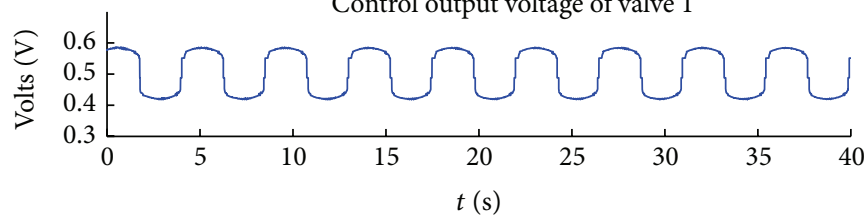

Control output voltage of valve 2

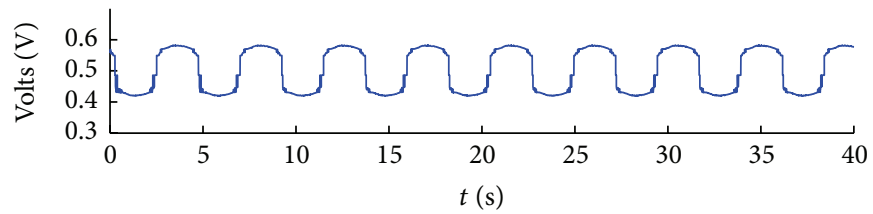

Control output voltage of valve 3

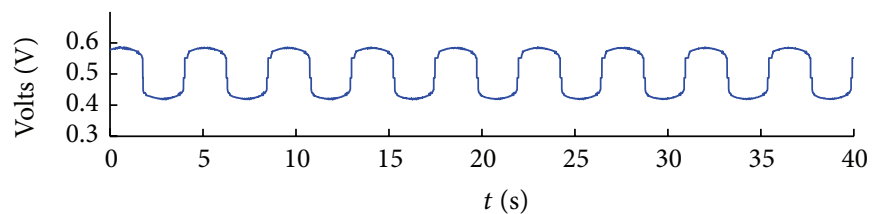

FIGURE 10: Joint space tracking error control voltages for Experiment 1.3.
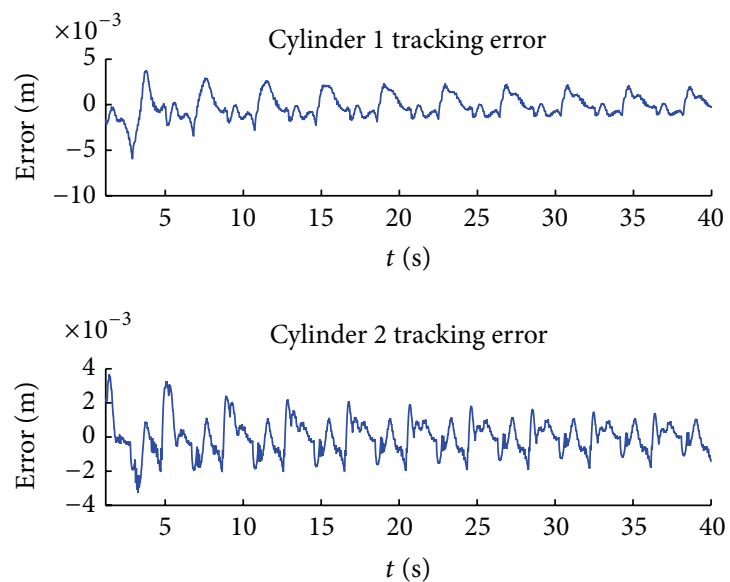

Cylinder 3 tracking error
Control output voltage of valve 1

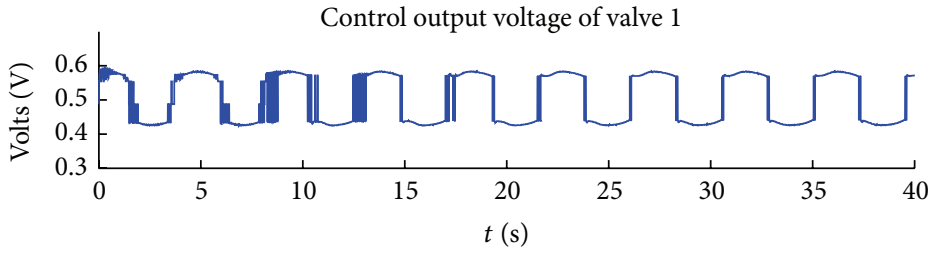

Control output voltage of valve 2

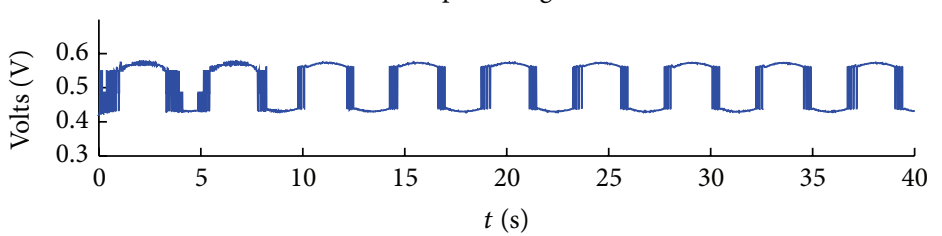

Control output voltage of valve 3

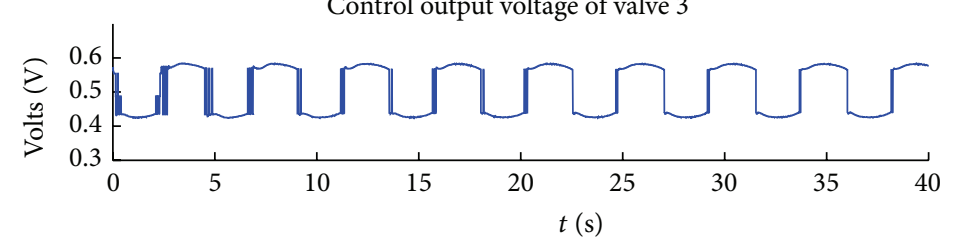

FIGURE 11: Joint space tracking error and control voltages for Experiment 2.1.

TABLE 3: Joint space tracking experiments error analysis (unit: $\mathrm{mm}$ ).

\begin{tabular}{lccccccccc}
\hline \multirow{2}{*}{ Axis } & \multicolumn{2}{c}{ Exp. 1.1 } & \multicolumn{2}{c}{ Exp. 1.2 } & \multicolumn{2}{c}{ Exp. 1.3 } & \multicolumn{3}{c}{ Exp. 2.1 } \\
& $e_{F}$ & $e_{\text {rms }}$ & $e_{F}$ & $e_{\text {rms }}$ & $e_{F}$ & $e_{\text {rms }}$ & $e_{F}$ & $e_{\text {rms }}$ & $e_{\text {rel }}(\%)$ \\
\hline Cy1 & 7.76 & 3.01 & 8.06 & 2.57 & 1.65 & 0.76 & 2.27 & 0.95 \\
Cy2 & 6.74 & 2.48 & 6.02 & 2.01 & 1.94 & 0.85 & 1.88 & 0.73 \\
Cy3 & 4.66 & 2.02 & 6.76 & 2.09 & 2.14 & 0.93 & 1.65 & 0.75 & 0.81 \\
\hline
\end{tabular}



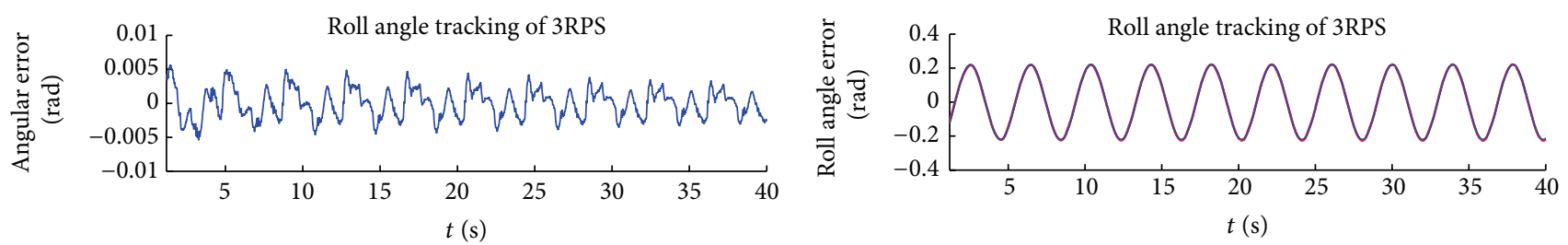

- Measured

Desired
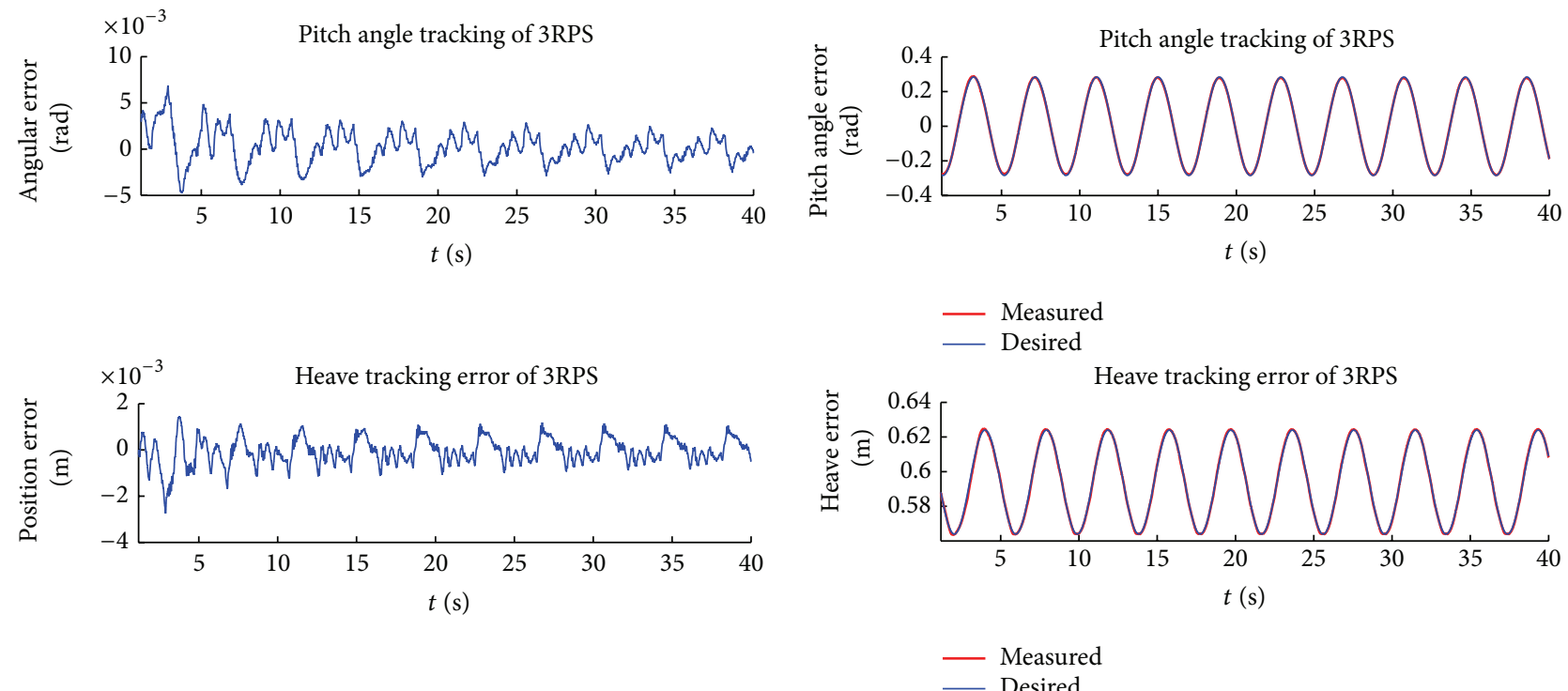

FIGURE 12: Workspace tracking error for Experiment 2.1.

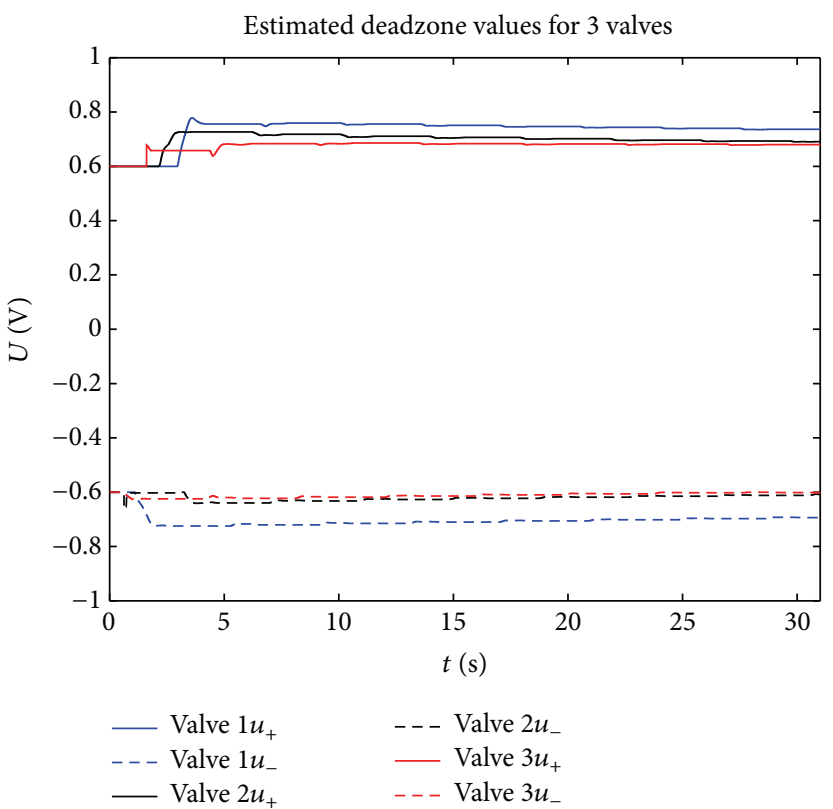

Figure 13: Deadzone estimation results for Experiment 2.1. 
TABLE 4: Workspace tracking experiments error analysis.

\begin{tabular}{lccccc}
\hline \multirow{2}{*}{ Axis } & & Exp. 2.1 & & \multicolumn{2}{c}{ Exp. 2.2} \\
& $e_{F}$ & $e_{\text {rms }}$ & $e_{\text {rel }}$ & $e_{F}$ & $e_{\text {rms }}$ \\
\hline Roll & $0.0044 \mathrm{rad}$ & $0.0022 \mathrm{rad}$ & $0.79 \%$ & $0.0039 \mathrm{rad}$ & $0.0019 \mathrm{rad}$ \\
Pitch & $0.0039 \mathrm{rad}$ & $0.0017 \mathrm{rad}$ & $0.53 \%$ & $0.0046 \mathrm{rad}$ & $0.0021 \mathrm{rad}$ \\
Heave & $1.64 \mathrm{~mm}$ & $0.67 \mathrm{~mm}$ & $0.84 \%$ & $1.65 \mathrm{~mm}$ & $0.68 \%$ \\
\hline
\end{tabular}
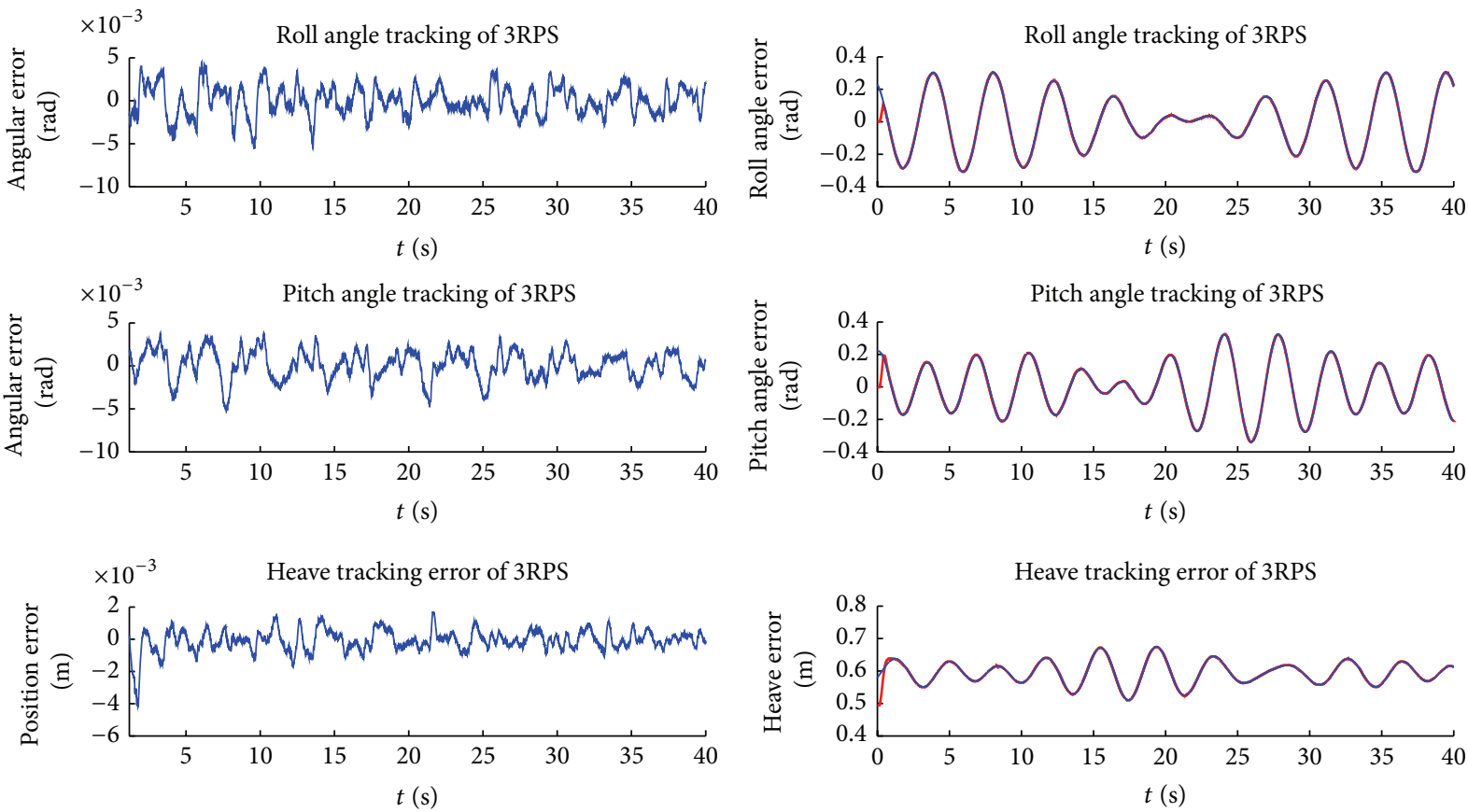

FIGURE 14: Workspace compound trajectory tracking experiment.

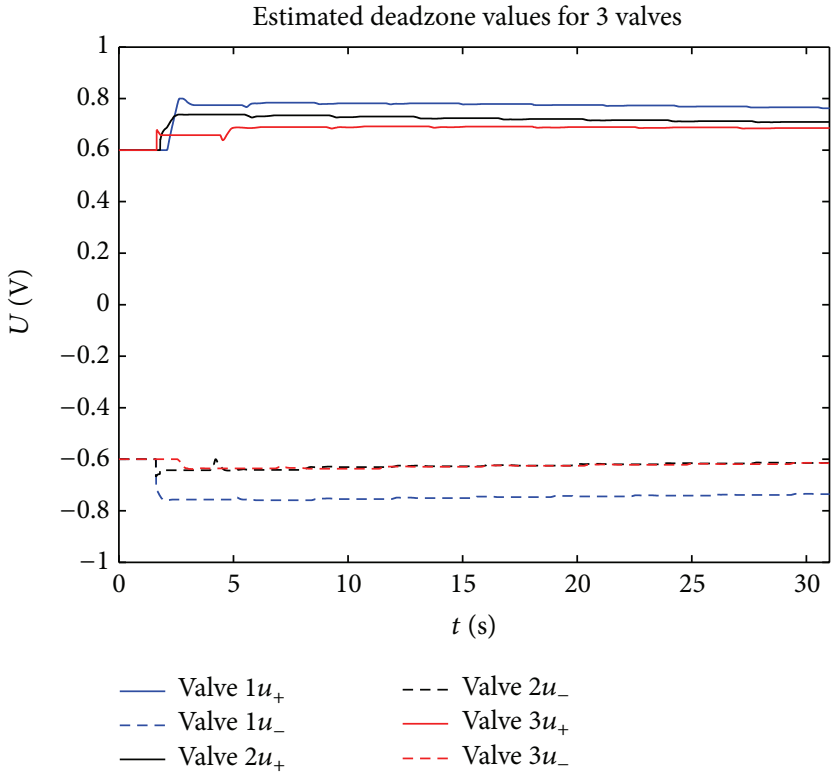

Figure 15: Deadzone estimation results for Experiment 2.2. 


\section{Appendix}

\section{Proof of Stability}

Define a Lyapunov function:

$$
V_{1}=\frac{1}{2} \mathbf{e}_{s}^{T} \mathbf{M}_{e} \mathbf{e}_{s} .
$$

The derivation of $V_{1}$ is

$$
\dot{V}_{1}=\mathbf{e}_{s}^{T}\left[\bar{A}_{a} \mathbf{p}_{L}+\boldsymbol{\varphi}_{a}^{T} \boldsymbol{\theta}_{a}+\tilde{\mathbf{f}}_{0}-\mathbf{M}_{e} \ddot{\mathbf{x}}_{d}+\mathbf{M}_{e} \mathbf{X e}\right] .
$$

Substitute (22) and (23) into (A.2):

$$
\begin{aligned}
\dot{V}_{1}= & \bar{A}_{a} \mathbf{e}_{s}^{T} \mathbf{e}_{p}-\mathbf{e}_{s}^{T} \mathbf{K}_{p} \mathbf{e}_{s} \\
& +\mathbf{e}_{s}^{T}\left[\bar{A}_{a} \mathbf{p}_{L d a 2}+\bar{A}_{a} \mathbf{p}_{L d s 2}-\boldsymbol{\varphi}_{a}^{T} \widetilde{\boldsymbol{\theta}}_{a}+\widetilde{\mathbf{f}}_{0}\right]
\end{aligned}
$$

According to the definition of $\mathbf{p}_{L d}$,

$$
\begin{aligned}
\dot{V}_{1}= & \bar{A}_{a} \mathbf{e}_{s}^{T} \mathbf{e}_{p}-\mathbf{e}_{s}^{T} \mathbf{K}_{p} \mathbf{e}_{s} \\
& +\mathbf{e}_{s}^{T}\left[\bar{A}_{a} \mathbf{p}_{L d s 2}-\widetilde{\mathbf{d}}_{c 1}+\mathbf{R}_{1}(\mathbf{t})\right] .
\end{aligned}
$$

According to (25), $\mathbf{p}_{L d s 2}$ satisfies

$$
\begin{aligned}
\mathbf{e}_{s}^{T}\left[\bar{A}_{a} \mathbf{p}_{L d s 2}-\widetilde{\mathbf{d}}_{c 1}+\mathbf{R}_{1}(t)\right] & \leq \eta_{1} \\
\bar{A}_{a} \mathbf{e}_{s}^{T} \mathbf{p}_{L d s 2} & \leq 0 .
\end{aligned}
$$

Then, the derivation of $V_{1}$ can be rewritten in the form

$$
\dot{V}_{1} \leq \bar{A}_{a} \mathbf{e}_{s}^{T} \mathbf{e}_{p}-\mathbf{e}_{s}^{T} \mathbf{K}_{p} \mathbf{e}_{s}+\eta_{1} .
$$

Define another Lyapunov function $V_{2}$ :

$$
V_{2}=V_{1}+\frac{1}{2} \mathbf{e}_{p}^{T} \mathbf{e}_{p}
$$

As in the previous step, derive $V_{2}$

$$
\begin{aligned}
\dot{V}_{2}= & \left.\dot{V}_{1}\right|_{\mathbf{p}_{L d}}-\mathbf{e}_{p}^{T} \mathbf{K}_{q} \mathbf{e}_{p} \\
& +\mathbf{e}_{p}^{T}\left[\mathbf{q}_{L d a 2}+\mathbf{q}_{L d s 2}+\mathbf{q}_{L g} \boldsymbol{\Delta}_{D}-\boldsymbol{\varphi}_{b}^{T} \widetilde{\boldsymbol{\theta}}_{b}+\widetilde{\mathbf{d}}_{0}\right] .
\end{aligned}
$$

Based on the definition of $\mathbf{q}_{L d}, \dot{V}_{2}$ is expressed as

$$
\dot{V}_{2}=\left.\dot{V}_{1}\right|_{\mathbf{p}_{L d}}-\mathbf{e}_{p}^{T} \mathbf{K}_{q} \mathbf{e}_{p}+\mathbf{e}_{p}^{T}\left[\mathbf{q}_{L d s 2}-\widetilde{\mathbf{d}}_{c 2}+\mathbf{R}_{2}(\mathbf{t})\right] .
$$

According to (32), $\mathbf{q}_{L d s 2}$ satisfies

$$
\begin{array}{r}
\mathbf{e}_{p}^{T}\left[\mathbf{q}_{L d s 2}-\tilde{\mathbf{d}}_{c 2}+\mathbf{R}_{2}(\mathbf{t})\right] \leq \eta_{2} \\
\mathbf{e}_{p}^{T} \mathbf{q}_{L d s 2} \leq 0
\end{array}
$$

which means

$$
\dot{V}_{2} \leq-\mathbf{e}_{s}^{T} \mathbf{K}_{p} \mathbf{e}_{s}-\mathbf{e}_{p}^{T} \mathbf{K}_{q} \mathbf{e}_{p}+\eta_{1}+\eta_{2} \leq-\lambda V_{2}+\eta,
$$

where $\lambda=\min \left\{2 \mathbf{K}_{q}(i), 2 \mathbf{K}_{p m}(i)\right\}, \mathbf{K}_{p m}=\mathbf{K}_{p} / \mathbf{M}_{e}$, as $\mathbf{K}_{q}, \mathbf{K}_{p}$, and $\mathbf{M}_{e}$ are all diagonal matrix, $\mathbf{K}_{p m}$ is also diagonal, and $i$ stands for the element of $i$ th row and $i$ th column in each matrix.

The differential function (A.11) has the solution of the form

$$
\dot{V}_{2}(t) \leq e^{-\lambda t} V_{2}(0)+\eta\left(1-e^{-\lambda t}\right) \lambda^{-1}
$$

which indicates that the up-bound of $\mathbf{e}_{L}=\left[e_{s m}, e_{p m}\right]^{T}$ can be expressed as

$$
\left\|\mathbf{e}_{L}\right\|^{2} \leq e^{-\lambda t}\left\|\mathbf{e}_{L}(\mathbf{0})\right\|+2 \eta\left(1-e^{-\lambda t}\right) \lambda^{-1},
$$

where $e_{s m}=\left\|\mathbf{e}_{s}\right\|$ and $e_{p m}=\left\|\mathbf{e}_{p}\right\|$. From (A.12), the error vector $\mathbf{e}$ is exponentially convergent to a spherical domain. The size of the domain can be adjusted by changing $K_{p}, K_{q}$, $\eta_{1}$, and $\eta_{2}$, which indicates error vector control.

According to Lyapunov theory, $\dot{V}_{2}$ gradually converges into a spherical area, and the size is controlled by the value of $\mathbf{K}_{p}, \mathbf{K}_{q}, \eta_{1}$, and $\eta_{2}$; thus, the tracking error vector $\mathbf{e}$ is always bounded, and the controller designed in Section 3 is stable.

\section{Additional Points}

The English in this document has been checked by at least two professional editors, both native speakers of English. For a certificate, please see: http://www.textcheck.com/certificate/2RlfI1.

\section{Competing Interests}

The authors declare that there is no conflict of interests regarding the publication of this paper.

\section{Acknowledgments}

This work is supported by National Natural Science Foundation of China (no. 51375430).

\section{References}

[1] J.-P. Merlet, Parallel Robots, Kluwer Academic Publishers, Norwell, Mass, USA, 2002.

[2] P. Wos and R. Dindorf, "Synchronized trajectory tracking control of 3-DoF hydraulic translational parallel manipulator," Advances in Intelligent Systems and Computing, vol. 317, pp. 269277, 2015.

[3] M. Ramsauer, M. Kastner, P. Ferrara, R. Naderer, and H. Gattringer, "A pneumatically driven stewart platform used as fault detection device," Applied Mechanics and Materials, vol. 186, pp. 227-233, 2012.

[4] B. Andrievsky, D. Kazunin, D. Kostygova et al., "Control of pneumatically actuated 6-dof stewart platform for driving simulator," in Proceedings of the 19th International Conference on Methods and Models in Automation and Robotics (MMAR '14), pp. 663-668, Miedzyzdroje, Poland, September 2014.

[5] T. Kimura, S. Hara, T. Fujita, and T. Kagawa, "Feedback linearization for pneumatic actuator systems with static friction," Control Engineering Practice, vol. 5, no. 10, pp. 1385-1394, 1997. 
[6] R. Richardson, A. R. Plummer, and M. D. Brown, "Self-tuning control of a low-friction pneumatic actuator under the influence of gravity," IEEE Transactions on Control Systems Technology, vol. 9, no. 2, pp. 330-334, 2001.

[7] H. Schulte and H. Hahn, "Fuzzy state feedback gain scheduling control of servo-pneumatic actuators," Control Engineering Practice, vol. 12, no. 5, pp. 639-650, 2004.

[8] S. Ning and G. M. Bone, "Experimental comparison of two pneumatic servo position control algorithms," in Proceedings of the IEEE International Conference on Mechatronics and Automation (ICMA '05), vol. 1, pp. 37-42, IEEE, August 2005.

[9] A. Girin, F. Plestan, X. Brun, and A. Glumineau, "Highorder sliding-mode controllers of an electropneumatic actuator: application to an aeronautic benchmark," IEEE Transactions on Control Systems Technology, vol. 17, no. 3, pp. 633-645, 2009.

[10] C. Guan and S. Pan, "Adaptive sliding mode control of electrohydraulic system with nonlinear unknown parameters," Control Engineering Practice, vol. 16, no. 11, pp. 1275-1284, 2008.

[11] B. Yao and M. Tomizuka, "Adaptive robust control of SISO nonlinear systems in a semi-strict feedback form," Automatica, vol. 33, no. 5, pp. 893-900, 1997.

[12] M. Smaoui, X. Brun, and D. Thomasset, "A study on tracking position control of an electropneumatic system using backstepping design," Control Engineering Practice, vol. 14, no. 8, pp. 923933, 2006.

[13] D. Meng, G. Tao, and X. Zhu, "Integrated direct/indirect adaptive robust motion trajectory tracking control of pneumatic cylinders," International Journal of Control, vol. 86, no. 9, pp. 1620-1633, 2013.

[14] K. Grewal, R. Dixon, and J. Pearson, "Control design for a pneumatically actuated parallel link manipulator," in Proceedings of the 21st International Conference on Systems Engineering (ICSEng '11), pp. 43-48, Las Vegas, Nev, USA, August 2011.

[15] K. S. Grewal, R. Dixon, and J. Pearson, "LQG controller design applied to a pneumatic stewart-gough platform," International Journal of Automation and Computing, vol. 9, no. 1, pp. 45-53, 2012.

[16] J. Pradipta, M. Klunder, M. Weickgenannt, and O. Sawodny, "Development of a pneumatically driven flight simulator Stewart platform using motion and force control," in Proceedings of the IEEE/ASME International Conference on Advanced Intelligent Mechatronics (AIM '13), pp. 158-163, Wollongong, Australia, July 2013.

[17] A. C. Valdiero, D. Bavaresco, and P. L. Andrighetto, "Experimental identification of the dead zone in proportional directional pneumatic valves," International Journal of Fluid Power, vol. 9, no. 1, pp. 27-33, 2008.

[18] A. C. Valdiero, C. S. Ritter, C. F. Rios, and M. Rafikov, "Nonlinear mathematical modeling in pneumatic servo position applications," Mathematical Problems in Engineering, vol. 2011, Article ID 472903, 16 pages, 2011.

[19] A. Mohanty and B. Yao, "Integrated direct/indirect adaptive robust control of hydraulic manipulators with valve deadband," IEEE/ASME Transactions on Mechatronics, vol. 16, no. 4, pp. 707-715, 2011.

[20] X.-S. Wang, C.-Y. Su, and H. Hong, "Robust adaptive control of a class of nonlinear systems with unknown dead-zone," Automatica, vol. 40, no. 3, pp. 407-413, 2004.

[21] G. Tao and P. V. Kokotovic, "Adaptive control of plants with unknown dead-zones," IEEE Transactions on Automatic Control, vol. 39, no. 1, pp. 59-68, 1994.
[22] F. Bu and B. Yao, "Adaptive robust precision motion control of single-rod hydraulic actuators with time-varying unknown inertia: a case study," in Proceedings of the ASME International Mechanical Engineering Congress and Exposition (IMECE '99), vol. 6, pp. 131-138, FPST, Nashville, Tenn, USA, November 1999.

[23] C. Hu, B. Yao, and Q. Wang, "Adaptive robust precision motion control of systems with unknown input dead-zones: a case study with comparative experiments," IEEE Transactions on Industrial Electronics, vol. 58, no. 6, pp. 2454-2464, 2011.

[24] J. H. Pérez-Cruz, E. Ruiz-Velázquez, J. De Jesús Rubio, and C. A. De Alba-Padilla, "Robust adaptive neurocontrol of SISO nonlinear systems preceded by unknown deadzone," Mathematical Problems in Engineering, vol. 2012, Article ID 342739, 23 pages, 2012.

[25] L. D. dos Santos Coelho and M. A. B. Cunha, "Adaptive cascade control of a hydraulic actuator with an adaptive deadzone compensation and optimization based on evolutionary algorithms," Expert Systems with Applications, vol. 38, no. 10, pp. 12262-12269, 2011.

[26] B. Magyar, C. Hős, and G. Stépán, “Influence of control valve delay and dead zone on the stability of a simple hydraulic positioning system," Mathematical Problems in Engineering, vol. 2010, Article ID 349489, 15 pages, 2010.

[27] P. L. Andrighetto, A. C. Valdiero, and D. Bavaresco, "Dead zone compensation in pneumatic servo systems," in Proceedings of the ABCM Symposium Series in Mechatronics, vol. 3, pp. 501-509, 2008.

[28] D. Meng, G. Tao, J. Chen, and W. Ban, "Modeling of a pneumatic system for high-accuracy position control," in Proceedings of the International Conference on Fluid Power and Mechatronics (FPM '11), pp. 505-510, IEEE, Hangzhou, China, August 2011.

[29] J. F. Carneiro and F. G. de Almeida, "Heat transfer evaluation of industrial pneumatic cylinders," Proceedings of the Institution of Mechanical Engineers. Part I, vol. 221, no. 1, pp. 119-128, 2007.

[30] B. Yao, "High performance adaptive robust control of nonlinear systems: a general framework and new schemes," in Proceedings of the 36th IEEE Conference on Decision and Control, vol. 3, pp. 2489-2494, IEEE, San Diego, Calif, USA, December 1997.

[31] D. Sun, R. Lu, J. K. Mills, and C. Wang, "Synchronous tracking control of parallel manipulators using cross-coupling approach," The International Journal of Robotics Research, vol. 25, no. 11, pp. 1137-1147, 2006.

[32] D. Sun, C. Wang, W. Shang, and G. Feng, "A synchronization approach to trajectory tracking of multiple mobile robots while maintaining time-varying formations," IEEE Transactions on Robotics, vol. 25, no. 5, pp. 1074-1086, 2009. 


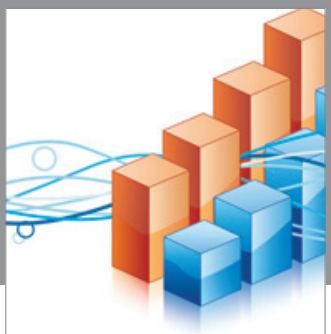

Advances in

Operations Research

vatem alat4

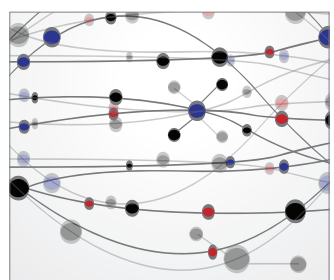

\section{The Scientific} World Journal
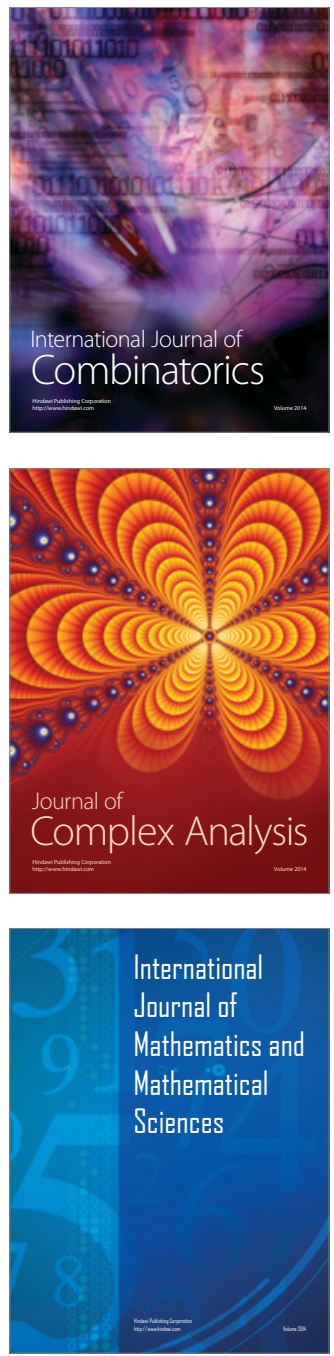
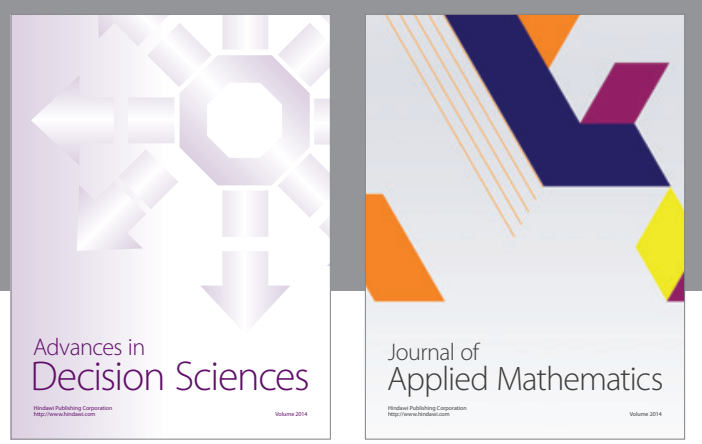

Algebra

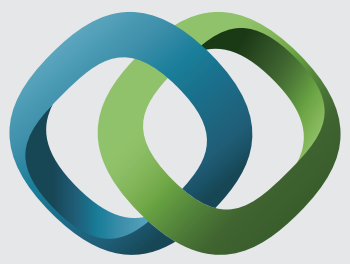

\section{Hindawi}

Submit your manuscripts at

http://www.hindawi.com
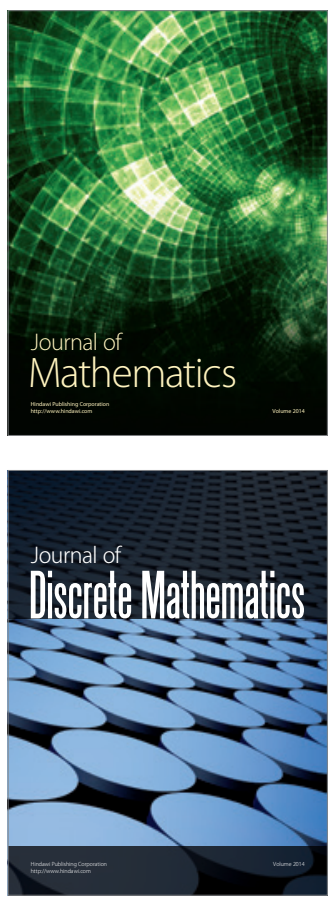

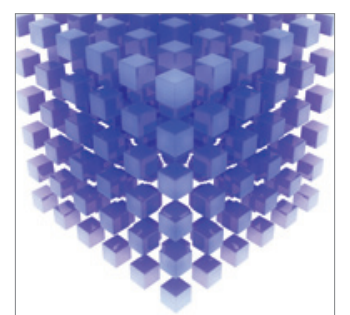

Mathematical Problems in Engineering
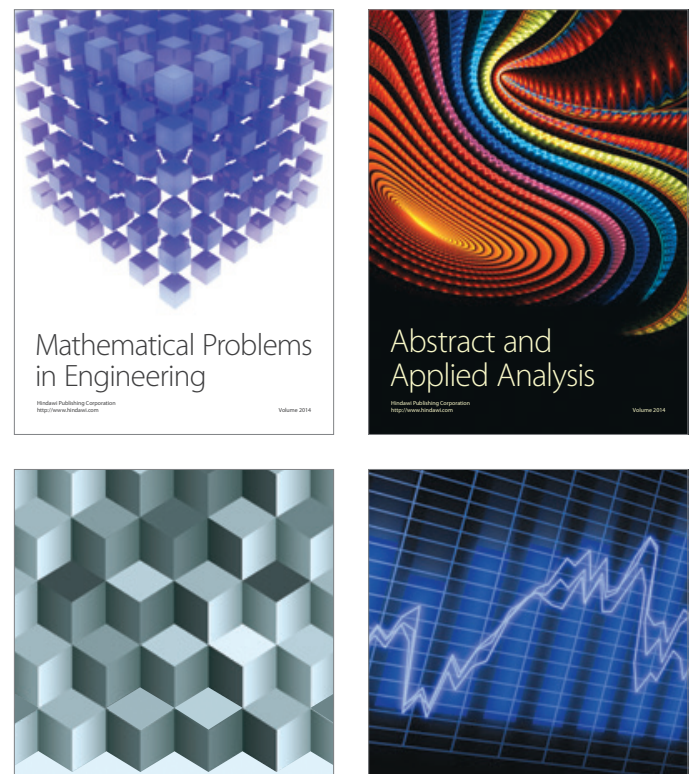

Journal of

Function Spaces

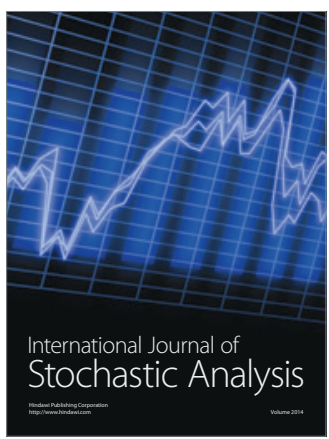

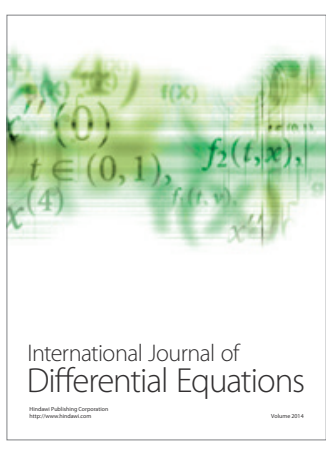
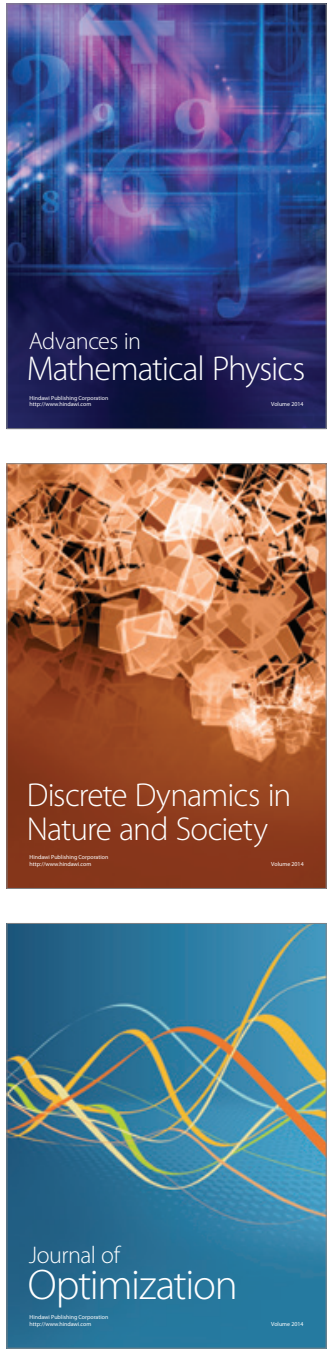